\title{
A Maximum Principle for Controlled Time-Symmetric Forward-Backward Doubly Stochastic Differential Equation with Initial-Terminal Sate Constraints
}

\author{
Shaolin Ji, ${ }^{1}$ Qingmeng Wei, ${ }^{2}$ and Xiumin Zhang ${ }^{2}$ \\ ${ }^{1}$ Institute for Financial Studies and Institute of Mathematics, Shandong University, \\ Shandong, Jinan 250100, China \\ ${ }^{2}$ Institute of mathematics, Shandong University, Shandong, Jinan 250100, China \\ Correspondence should be addressed to Shaolin Ji, jsl@sdu.edu.cn
}

Received 2 October 2012; Accepted 15 November 2012

Academic Editor: Jen-Chih Yao

Copyright (C) 2012 Shaolin Ji et al. This is an open access article distributed under the Creative Commons Attribution License, which permits unrestricted use, distribution, and reproduction in any medium, provided the original work is properly cited.

We study the optimal control problem of a controlled time-symmetric forward-backward doubly stochastic differential equation with initial-terminal state constraints. Applying the terminal perturbation method and Ekeland's variation principle, a necessary condition of the stochastic optimal control, that is, stochastic maximum principle, is derived. Applications to backward doubly stochastic linear-quadratic control models are investigated.

\section{Introduction}

It is well known that general coupled forward-backward stochastic differential equations (FBSDEs) consist of a forward SDE of Itô's type and a backward SDE of Pardoux-Peng's (see $[1,2])$. Since Antonelli [3] first studied FBSDEs in early 1990s, FBSDEs have been studied widely in many papers (see [4-7]). FBSDEs are often encountered in the optimization problem when applying stochastic maximum principle (see $[8,9]$ ). In finance, FBSDEs are used when considering problems with the large investors; see $[6,10,11]$. Such equations are also used in the potential theory (see [12]). Moreover, one can apply FBSDEs to study Homogenization and singular perturbation of certain quasilinear parabolic PDEs with periodic structures (see $[13,14]$ ).

In order to produce a probabilistic representation of certain quasilinear stochastic partial differential equations (SPDEs), Pardoux and Peng [15] first introduced backward doubly stochastic differential equations (BDSDEs) and proved the existence and uniqueness 
theorem of BDSDEs. Using such BDSDEs they proved the existence and uniqueness theorem of those quasilinear SPDEs and thus significantly extended the famous Feynman-Kac formula for such SPDEs.

Peng and Shi [16] studied the following time-symmetric forward-backward doubly stochastic differential equations (FBDSDEs):

$$
\begin{gathered}
-d x_{t}=F\left(t, x_{t}, z_{t}, y_{t}, q_{t}, u_{t}\right) d t+G\left(t, x_{t}, z_{t}, y_{t}, q_{t}, u_{t}\right) d W_{t}-z_{t} d B_{t}, \quad 0 \leq t \leq T, \\
x_{0}=\xi, \\
-d y_{t}=f\left(t, x_{t}, z_{t}, y_{t}, q_{t}, u_{t}\right) d t+g\left(t, x_{t}, z_{t}, y_{t}, q_{t}, u_{t}\right) d B_{t}-q_{t} d W_{t}, \quad 0 \leq t \leq T, \\
y_{T}=\eta,
\end{gathered}
$$

which generalized the general FBSDEs. Here the forward equation is "forward" with respect to a standard stochastic integral $d W_{t}$, as well as "backward" with respect to a backward stochastic integral $d B_{t}$; the coupled "backward equation" is "forward" under the backward stochastic integral $d B_{t}$ and "backward" under the forward one. In other words, both the forward equation and the backward one are BDSDEs with different directions of stochastic integral. Under certain monotonicity conditions, they proved the uniqueness and existence theorem for these equations. In [17], when deriving the stochastic maximum principle of backward doubly stochastic optimal control problems, Han et al. showed that this kind of equations are just the state equation and adjoint equation of their optimal control problem.

In this paper, we study a stochastic optimal control problem with initial-terminal state constraints where the controlled system is described by the above time-symmetric FBDSDEs. We suppose that the initial state $\xi$ and the terminal state $\eta$ fall in two convex sets, respectively, and the corresponding states $x_{T}^{(\xi, \eta, u(\cdot))}$ and $y_{0}^{(\xi, \eta, u(\cdot))}$ satisfy the constraints $E\left(\psi\left(x_{T}^{(\xi, \eta, u(\cdot))}\right)\right)=a$ and $E\left(h\left(y_{0}^{(\xi, \eta, u(\cdot))}\right)\right)=b$, respectively. Then we minimize the following cost function:

$$
J(\xi, \eta, u(\cdot)) \triangleq E\left[\int_{0}^{T} l(x(t), z(t), y(t), q(t), u(t), t) d t+\chi(\xi)+\lambda(\eta)+\phi(x(T))+\gamma(y(0))\right]
$$

It is well known that the maximum principle is an important approach to study optimal control problems. The systematic account of this theory can be found in $[9,18]$. When the controlled system under consideration is assumed to be with state constraints, especially with sample-wise constraints, the corresponding stochastic optimal control problems are difficult to solve. A sample-wise constraint requires that the state be in a given set with probability 1, for example, a nonnegativity constraint on the wealth process, that is, bankruptcy prohibition in financial markets. In order to deal with such optimal control problems, an approach named "terminal perturbation method" was introduced and applied in financial optimization problems recently (see [19-22]). This method is based on the dual method or martingale method introduced by Bielecki et al. in [23] and El Karoui et al. in [24]. It mainly applies Ekeland's variational principle to tackle the state constraints and derive a stochastic maximum principle which characterizes the optimal solution. For other works about the optimization problem with state constraints, the readers may refer to $[25,26]$. In this paper, 
a stochastic maximum principle is obtained for the controlled time-symmetric FBDSDEs with initial-terminal state constraints by using Ekeland's variational principle.

We give three specific applications to illustrate our theoretical results. In the first application, the controlled state equations are composed of a normal FSDE and a BDSDE. By introducing a backward formulation of the controlled system (inspired by [21]), we present the stochastic maximum principle for the optimal control. As a special case, we only consider one BDSDE as our state equation in the second application. As stated in the last application, our results can be applied in forward-backward doubly stochastic linear-quadratic (LQ) optimal control problems. The explicit expression of the optimal control is derived. Since the control system of SPDEs can be transformed to the relevant control system of FBDSDEs, our results can be used to solve the optimal control problem of one kind of SPDEs.

This paper is organized as follows. In Section 2.1, we recall some preliminaries. And we formulate our control problem in Section 2.2. In Section 2.3, by applying Ekeland's variation principle we obtain a stochastic maximum principle of this controlled timesymmetric FBDSDEs with initial-terminal state constraints. Some applications are given in the last section.

\section{The Main Problem}

\subsection{Preliminaries}

Let us first recall the existence and uniqueness results of the BDSDE which was introduced by Pardoux and Peng [15] and an extension of the well-known Itô's formula which would be often used in this paper.

Let $(\Omega, \mathcal{F}, P)$ be a probability space, and let $T>0$ be fixed throughout this paper. Let $\left\{\mathbf{W}_{t}, 0 \leq t \leq T\right\}$ and $\left\{\mathbf{B}_{t}, 0 \leq t \leq T\right\}$ be two mutually independent standard Brownian motion processes, with values in $\mathbb{R}^{d}, \mathbb{R}^{l}$, respectively, defined on $(\Omega, \mathcal{F}, P)$. Let $\mathbb{N}$ denote the class of $P$-null set of $\mathcal{F}$. For each $t \in 0, T]$, we define $\mathcal{F}_{t} \triangleq \mathcal{F}_{t}^{W} \vee \mathcal{F}_{t, T}^{B}$, where

$$
\mathcal{F}_{t}^{W}=\mathcal{F}_{0, t}^{W}=\sigma\left\{W_{r}-W_{0} ; 0 \leq r \leq t\right\} \vee \mathbb{N}, \quad \mathcal{F}_{t}^{B}=\mathcal{F}_{0, t}^{B}=\sigma\left\{B_{r}-B_{t} ; t \leq r \leq T\right\} \vee \mathbb{N} .
$$

Note that the collection $\left\{\mathcal{F}_{t}, t \in[0, T]\right\}$ is neither increasing nor decreasing, and it does not constitute a filtration.

For any Euclidean space $H$, we denote by $\langle\cdot, \cdot\rangle$ the scale product of $H$. The Euclidean norm of a vector $y \in \mathbb{R}^{k}$ will be denoted by $|y|$, and for a $d \times n$ matrix $\mathrm{A}$, we define $\|A\|=$ $\sqrt{\operatorname{Tr}\left(A A^{*}\right)}$.

For any $n \in N$, let $M^{2}\left(0, T ; \mathbb{R}^{n}\right)$ denote the set of (classes of $d P \otimes d t$ a.e. equal) $n$ dimensional jointly measurable stochastic processes $\left\{\varphi_{t} ; t \in[0, T]\right\}$ which satisfy

(i) $E \int_{0}^{T}\left|\varphi_{t}\right|^{2} d t<\infty$; (ii) $\varphi_{t}$ is $\mathcal{F}_{t}$-measurable, for a.e. $t \in[0, T]$.

We denote by $S^{2}\left(0, T ; \mathbb{R}^{n}\right)$ the set of continuous $n$-dimensional stochastic processes which satisfy:

(i) $E\left(\sup _{0 \leq t \leq T}\left|\varphi_{t}\right|^{2}\right)<\infty$; (ii) $\varphi_{t}$ is $\mathcal{F}_{t}$-measurable, for any $t \in[0, T]$.

Let

$$
f: \Omega \times[0, T] \times \mathbb{R}^{k} \times \mathbb{R}^{k \times d} \longrightarrow \mathbb{R}^{k}, \quad g: \Omega \times[0, T] \times \mathbb{R}^{k} \times \mathbb{R}^{k \times d} \longrightarrow \mathbb{R}^{k \times l}
$$

be jointly measurable such that for any $(y, q) \in \mathbb{R}^{k} \times \mathbb{R}^{k \times d}, f(\cdot, y, q) \in M^{2}\left(0, T ; \mathbb{R}^{k}\right), g(\cdot, y, q) \in$ $M^{2}\left(0, T ; \mathbb{R}^{k \times l}\right)$. 
Moreover, we assume that there exist constants $C>0$ and $0<\alpha<1$ such that for any $(\omega, t) \in \Omega \times[0, T],\left(y_{1}, q_{1}\right),\left(y_{2}, q_{2}\right) \in \mathbb{R}^{k} \times \mathbb{R}^{k \times l}$,

$$
\begin{aligned}
& \left|f\left(t, y_{1}, q_{1}\right)-f\left(t, y_{2}, q_{2}\right)\right|^{2} \leq C\left(\left|y_{1}-y_{2}\right|^{2}+\left\|q_{1}-q_{2}\right\|^{2}\right) \\
& \left\|g\left(t, y_{1}, q_{1}\right)-g\left(t, y_{2}, q_{2}\right)\right\|^{2} \leq C\left|y_{1}-y_{2}\right|^{2}+\alpha\left\|q_{1}-q_{2}\right\|^{2} .
\end{aligned}
$$

Given $\eta \in L^{2}\left(\Omega, \mathcal{F}_{T}, P ; \mathbb{R}^{k}\right)$, we consider the following BDSDE:

$$
y_{t}=\eta+\int_{t}^{T} f\left(s, y_{s}, q_{s}\right) d s+\int_{t}^{T} g\left(s, y_{s}, q_{s}\right) d B_{s}-\int_{t}^{T} q_{s} d W_{s}, \quad 0 \leq t \leq T
$$

We note that the integral with respect to $\left\{B_{t}\right\}$ is a "backward Itô integral" and the integral with respect to $\left\{W_{t}\right\}$ is a standard forward Itô integral. These two types of integrals are particular cases of the Itô-Skorohod integral; see Nualart and Pardoux [27].

By Theorem 1.1 in [15], (2.3) has a unique solution $(y, q) \in S^{2}\left(0, T ; \mathbb{R}^{k}\right) \times$ $M^{2}\left(0, T ; \mathbb{R}^{k \times d}\right)$.

Next let us recall an extension of the well-known Itô's formula in [21] which would be often used in this paper.

Lemma 2.1. Let $\alpha \in S^{2}\left(0, T ; \mathbb{R}^{k}\right), \beta \in M^{2}\left(0, T ; \mathbb{R}^{k}\right), \gamma \in M^{2}\left(0, T ; \mathbb{R}^{k \times l}\right), \delta \in M^{2}\left(0, T ; \mathbb{R}^{k \times d}\right)$ be such that

$$
\alpha_{t}=\alpha_{0}+\int_{0}^{t} \beta_{s} d s+\int_{0}^{t} \gamma_{s} d B_{s}+\int_{0}^{t} \delta_{s} d W_{s}, \quad 0 \leq t \leq T
$$

Then,

$$
\begin{gathered}
\left|\alpha_{t}\right|^{2}=\left|\alpha_{0}\right|^{2}+2 \int_{0}^{t}\left(\alpha_{s}, \beta_{s}\right) d s+2 \int_{0}^{t}\left(\alpha_{s}, \gamma_{s} d B_{s}\right)-\int_{0}^{t}\left\|r_{s}\right\|^{2} d s+\int_{0}^{t}\left\|\delta_{s}\right\|^{2} d s+2 \int_{0}^{t}\left(\alpha_{s}, \delta_{s} d W_{s}\right), \\
E\left|\alpha_{t}\right|^{2}=E\left|\alpha_{0}\right|^{2}+2 E \int_{0}^{t}\left(\alpha_{s}, \beta_{s}\right) d s-E \int_{0}^{t}\left\|r_{s}\right\|^{2} d s+E \int_{0}^{t}\left\|\delta_{s}\right\|^{2} d s .
\end{gathered}
$$

Generally, for $\phi \in C^{2}\left(\mathbb{R}^{k}\right)$,

$$
\begin{aligned}
\phi\left(\alpha_{t}\right)= & \phi\left(\alpha_{0}\right)+\int_{0}^{t}\left(\phi^{\prime}\left(\alpha_{s}\right), \beta_{s}\right) d s+\int_{0}^{t}\left(\phi^{\prime}\left(\alpha_{s}\right), \gamma_{s} d B_{s}\right)+\int_{0}^{t}\left(\phi^{\prime}\left(\alpha_{s}\right), \delta_{s} d W_{s}\right) \\
& -\frac{1}{2} \int_{0}^{t} \operatorname{Tr}\left[\phi^{\prime \prime}\left(\alpha_{s}\right) \gamma_{s} \gamma_{s}^{*}\right] d s+\frac{1}{2} \int_{0}^{t} \operatorname{Tr}\left[\phi^{\prime \prime}\left(\alpha_{s}\right) \delta_{s} \delta_{s}^{*}\right] d s
\end{aligned}
$$




\subsection{Problem Formulation}

Let $K$ be a nonempty convex subset of $\mathbb{R}^{n \times d}$. We set

$$
U[0, T]=\left\{u(\cdot) \mid u(t) \in K \text {, a.e., a.s., } 0 \leq t \leq T ; u(\cdot) \in M^{2}\left(0, T ; \mathbb{R}^{n \times d}\right)\right\}
$$

An element of $U[0, T]$ is called an admissible control. Now let

$$
\begin{gathered}
F: \Omega \times[0, T] \times \mathbb{R}^{n} \times \mathbb{R}^{n \times l} \times \mathbb{R}^{k} \times \mathbb{R}^{k \times d} \times \mathbb{R}^{n \times d} \longrightarrow \mathbb{R}^{n}, \\
G: \Omega \times[0, T] \times \mathbb{R}^{n} \times \mathbb{R}^{n \times l} \times \mathbb{R}^{k} \times \mathbb{R}^{k \times d} \times \mathbb{R}^{n \times d} \longrightarrow \mathbb{R}^{n \times d}, \\
f: \Omega \times[0, T] \times \mathbb{R}^{n} \times \mathbb{R}^{n \times l} \times \mathbb{R}^{k} \times \mathbb{R}^{k \times d} \times \mathbb{R}^{n \times d} \longrightarrow \mathbb{R}^{k}, \\
g: \Omega \times[0, T] \times \mathbb{R}^{n} \times \mathbb{R}^{n \times l} \times \mathbb{R}^{k} \times \mathbb{R}^{k \times d} \times \mathbb{R}^{n \times d} \longrightarrow \mathbb{R}^{k \times l}
\end{gathered}
$$

be jointly measurable such that for any $(x, z, y, q) \in \mathbb{R}^{n} \times \mathbb{R}^{n \times l} \times \mathbb{R}^{k} \times \mathbb{R}^{k \times d}$ and any $u(\cdot) \in U[0, T]$

$$
\begin{array}{ll}
F(\cdot, x, z, y, q, u(\cdot)) \in M^{2}\left(0, T ; \mathbb{R}^{n}\right), & G(\cdot, x, z, y, q, u(\cdot)) \in M^{2}\left(0, T ; \mathbb{R}^{n \times d}\right), \\
f(\cdot, x, z, y, q, u(\cdot)) \in M^{2}\left(0, T ; \mathbb{R}^{k}\right), & g(\cdot, x, z, y, q, u(\cdot)) \in M^{2}\left(0, T ; \mathbb{R}^{k \times l}\right) .
\end{array}
$$

Let

$$
\zeta(t)=(x(t), z(t), y(t), q(t))^{T}, \quad A(t, \zeta)=(-F,-G,-f,-g)^{T}(t, \zeta)
$$

We assume the following.

(H1) $\forall \zeta_{1}=\left(x_{1}, z_{1}, y_{1}, q_{1}\right), \zeta_{2}=\left(x_{2}, z_{2}, y_{2}, q_{2}\right) \in \mathbb{R}^{n} \times \mathbb{R}^{n \times l} \times \mathbb{R}^{k} \times \mathbb{R}^{k \times d}$ and $t \in[0, T]$, there exists a constant $\mu>0$ such that the following monotonicity condition holds for any $u(\cdot) \in U[0, T]:$

$$
\left\langle A\left(t, \zeta_{1}\right)-A\left(t, \zeta_{2}\right), \zeta_{1}-\zeta_{2}\right\rangle \leq-\mu\left|\zeta_{1}-\zeta_{2}\right|^{2}
$$

(H2) There exist constants $C>0$ and $0<\alpha<1 / 2$ such that for any $(\omega, t) \in \Omega \times[0, T]$, $u \in \mathbb{R}^{n \times d},\left(x_{1}, z_{1}, y_{1}, q_{1}\right),\left(x_{2}, z_{2}, y_{2}, q_{2}\right) \in \mathbb{R}^{n} \times \mathbb{R}^{n \times l} \times \mathbb{R}^{k} \times \mathbb{R}^{k \times d}$ the following conditions hold:

$$
\begin{aligned}
& \left|\vartheta\left(t, x_{1}, z_{1}, y_{1}, q_{1}, u\right)-\vartheta\left(t, x_{2}, z_{2}, y_{1}, q_{1}, u\right)\right|^{2} \\
& \quad \leq C\left(\left|x_{1}-x_{2}\right|^{2}+\left\|z_{1}-z_{2}\right\|^{2}+\left|y_{1}-y_{2}\right|^{2}+\left\|q_{1}-q_{2}\right\|^{2}\right), \\
& \left\|\varkappa\left(t, x_{1}, z_{1}, y_{1}, q_{1}, u\right)-\varkappa\left(t, x_{2}, z_{2}, y_{1}, q_{1}, u\right)\right\|^{2} \\
& \quad \leq C\left(\left|x_{1}-x_{2}\right|^{2}+\left|y_{1}-y_{2}\right|^{2}\right)+\alpha\left(\left\|z_{1}-z_{2}\right\|^{2}+\left\|q_{1}-q_{2}\right\|^{2}\right),
\end{aligned}
$$

where $\vartheta=(F, f), \varkappa=(G, g)$. 
(H3) $F, G, f, g, \psi, h, l, x, \lambda, \phi$ and $\gamma$ are continuous in their arguments and continuously differentiable in $(x, z, y, q, u)$, and the derivatives of $F, G, f, g$ in $(x, z, y, q, u)$ are bounded and $0<\left\|g_{z}(\cdot)\right\|<1 / 2,0<\left\|G_{z}(\cdot)\right\|<1 / 2,0<\left\|g_{q}(\cdot)\right\|<1 / 2,0<\left\|G_{q}(\cdot)\right\|<$ $1 / 2$; the derivatives of $l$ in $(x, y, z, q, u)$ are bounded by $C(1+|x|+|z|+|y|+|q|+\|u\|)$, and the derivatives of $\phi, x$, and $\psi$ in $x$ are bounded by $C(1+|x|) ; \gamma, \lambda$, and $h$ in $y$ are bounded by $C(1+|y|)$.

Given $\xi \in L^{2}\left(\Omega, \mathscr{F}_{0}, P ; \mathbb{R}^{n}\right), \eta \in L^{2}\left(\Omega, \mathcal{F}_{T}, P ; \mathbb{R}^{k}\right)$, and $\forall u(\cdot) \in U[0, T]$, let us consider the following time-symmetric FBDSDE:

$$
\begin{gathered}
-d x_{t}=F\left(t, x_{t}, z_{t}, y_{t}, q_{t}, u_{t}\right) d t+G\left(t, x_{t}, z_{t}, y_{t}, q_{t}, u_{t}\right) d W_{t}-z_{t} d B_{t}, \\
x_{0}=\xi, \\
-d y_{t}=f\left(t, x_{t}, z_{t}, y_{t}, q_{t}, u_{t}\right) d t+g\left(t, x_{t}, z_{t}, y_{t}, q_{t}, u_{t}\right) d B_{t}-q_{t} d W_{t} \\
y_{T}=\eta .
\end{gathered}
$$

Recall Theorem 2.2 in [16]. We have the following.

Theorem 2.2. For given $\xi \in L^{2}\left(\Omega, \mathcal{F}_{0}, P ; \mathbb{R}^{n}\right), \eta \in L^{2}\left(\Omega, \mathcal{F}_{T}, P ; \mathbb{R}^{k}\right)$ and $\forall u(\cdot) \in U[0, T]$, assume $(H 1) \sim(H 3)$; then (2.13) exists as a unique $\mathcal{F}_{t}$-adapted solution $(x(t), z(t), y(t), q(t))$.

In (2.13), we regard $\xi, \eta, u(\cdot)$ as controls. $\xi, \eta, u(\cdot)$ can be chosen from the following admissible set:

$$
\begin{aligned}
U=\left\{(\xi, \eta, u(\cdot)) \mid \xi \in K_{1} \subset \mathbb{R}^{n} \text { a.s., } \eta \in K_{2} \subset \mathbb{R}^{k} \text { a.s., } E\left[|\xi|^{2}\right]<\infty, E\left[|\eta|^{2}\right]\right. \\
<\infty, u(\cdot) \in U[0, T]\},
\end{aligned}
$$

where $K_{1}$ and $K_{2}$ are convex.

Remark 2.3. A main assumption in this paper is that the control domains are convex. For the terminal perturbation method, it is difficult to weaken or completely remove these assumptions. Until now, it remains an interesting and challenging open problem.

We also assume the state constraints

$$
E\left(\psi\left(x_{T}^{(\xi, \eta, u(\cdot))}\right)\right)=a, \quad E\left(h\left(y_{0}^{(\xi, \eta, u(\cdot))}\right)\right)=b
$$

For each $(\xi, \eta, u(\cdot)) \in U$, consider the following cost function:

$$
J(\xi, \eta, u(\cdot)) \triangleq E\left[\int_{0}^{T} l(x(t), z(t), y(t), q(t), u(t), t) d t+x(\xi)+\lambda(\eta)+\phi(x(T))+\gamma(y(0))\right] .
$$


Our optimization problem is

$$
\begin{gathered}
\inf _{(\xi, \eta, u(\cdot)) \in U} J(\xi, \eta, u(\cdot)) \\
\text { subject to } E\left(\psi\left(x_{T}^{(\xi, \eta, u(\cdot))}\right)\right)=a ; \quad E\left(h\left(y_{0}^{(\xi, \eta, u(\cdot))}\right)\right)=b .
\end{gathered}
$$

Definition 2.4. A triple of random variable $(\xi, \eta, u(\cdot)) \in U$ is called feasible for given $a \in \mathbb{R}^{n}$, $b \in \mathbb{R}^{k}$ if the solution (2.13) satisfies $E\left(\psi\left(x_{T}^{(\xi, \eta, u(\cdot))}\right)\right)=a$ and $E\left(h\left(y_{0}^{(\xi, \eta, u(\cdot))}\right)\right)=b$. We will denote by $\mathbb{N}(a, b)$ the set of all feasible $(\xi, \eta, u(\cdot))$ for any given $a$ and $b$. $\mathbb{N}(a, b)$.

A feasible $\left(\xi^{*}, \eta^{*}, u^{*}(\cdot)\right)$ is called optimal if it attains the minimum of $J(\xi, \eta, u(\cdot))$ over

The aim of this paper is to obtain a characterization of $\left(\xi^{*}, \eta^{*}, u^{*}(\cdot)\right)$, that is, the stochastic maximum principle.

\subsection{Stochastic Maximum Principle}

Using Ekeland's variational principle, we derive maximum principle for the optimization problem (2.17) in this section. For simplicity, we first study the case where $l(y(t), z(t)$, $y(t), q(t), u(t), t)=0, \chi(x)=0$ and $\lambda(y)=0$ in Sections 2.3.1-2.3.3 and then present the results for the general case in Section 2.3.4.

\subsubsection{Variational Equations}

For $\left(\xi^{1}, \eta^{1}, u^{1}(\cdot)\right),\left(\xi^{2}, \eta^{2}, u^{2}(\cdot)\right) \in U$, we define a metric in $U$ by

$$
\begin{aligned}
d\left(\xi^{1}, \eta^{1}, u^{1}(\cdot)\right),\left(\xi^{2}, \eta^{2}, u^{2}(\cdot)\right) \triangleq & \left(\left(E\left|\xi^{1}-\xi^{2}\right|^{2}\right)^{1 / 2}+E\left|\eta^{1}-\eta^{2}\right|^{2}\right)^{1 / 2} \\
& +\left(\left\|u^{1}(\cdot)-u^{2}(\cdot)\right\|^{2}\right)^{1 / 2}
\end{aligned}
$$

It is obvious that $(U, d(\cdot, \cdot))$ is a complete metric space.

Let $\left(\xi^{*}, \eta^{*}, u^{*}(\cdot)\right)$ be optimal and let $\left(x^{*}(\cdot), z^{*}(\cdot), y^{*}(\cdot), q^{*}(\cdot)\right)$ be the corresponding state processes of (2.13). for all $(\xi, \eta, u(\cdot)) \in U$ and for all $0 \leq \rho \leq 1$,

$$
\left(\xi^{\rho}, \eta^{\rho}, u^{\rho}(\cdot)\right) \triangleq\left(\xi^{*}+\rho\left(\xi-\xi^{*}\right), \quad \eta^{*}+\rho\left(\eta-\eta^{*}\right), u^{*}(\cdot)+\rho\left(u(\cdot)-u^{*}(\cdot)\right)\right) \in U .
$$

Let $\left(x^{\rho}(\cdot), z^{\rho}(\cdot), y^{\rho}(\cdot), q^{\rho}(\cdot)\right)$ be the state processes of (2.13) associated with $\left(\xi^{\rho}, \eta^{\rho}, u^{\rho}(\cdot)\right)$. 
To derive the first-order necessary condition, we let $(\widehat{x}(\cdot), \widehat{z}(\cdot), \widehat{y}(\cdot), \widehat{q}(\cdot))$ be the solution of the following time-symmetric FBDSDE:

$$
\begin{aligned}
-d \widehat{x}(t)= & \left(F_{x}^{*}(t) \widehat{x}(t)+F_{z}^{*}(t) \widehat{z}(t)+F_{y}^{*}(t) \widehat{y}(t)+F_{q}^{*}(t) \widehat{q}(t)+F_{u}^{*}(t)\left(u(\cdot)-u^{*}(\cdot)\right)\right) d t \\
& +\left(G_{x}^{*}(t) \widehat{x}(t)+G_{z}^{*}(t) \widehat{z}(t)+G_{y}^{*}(t) \widehat{y}(t)+G_{q}^{*}(t) \widehat{q}(t)+G_{u}^{*}(t)\left(u(\cdot)-u^{*}(\cdot)\right)\right) d W_{t} \\
& -\widehat{z}(t) d B(t), \\
& \widehat{x}(0)=\xi-\xi^{*}, \\
-d \widehat{y}(t)= & \left(f_{x}^{*}(t) \widehat{x}(t)+f_{z}^{*}(t) \widehat{z}(t)+f_{y}^{*}(t) \widehat{y}(t)+f_{q}^{*}(t) \widehat{q}(t)+f_{u}^{*}(t)\left(u(\cdot)-u^{*}(\cdot)\right)\right) d t \\
& +\left(g_{x}^{*}(t) \widehat{x}(t)+g_{z}^{*}(t) \widehat{z}(t)+g_{y}^{*}(t) \widehat{y}(t)+g_{q}^{*}(t) \widehat{q}(t)+g_{u}^{*}(t)\left(u(\cdot)-u^{*}(\cdot)\right)\right) d B_{t} \\
& -\widehat{q}(t) d W(t), \quad \\
& \widehat{y}(T)=\eta-\eta^{*},
\end{aligned}
$$

where $H_{k}^{*}(t)=H_{k}\left(t, x^{*}(\cdot), z^{*}(\cdot), y^{*}(\cdot), q^{*}(\cdot), u^{*}(\cdot)\right)$ for $H=F, G, f, g, k=x, z, y, q, u$, respectively. Equation (2.20) is called the variation equation.

Set

$$
\begin{array}{ll}
\tilde{x}_{\rho}(t)=\rho^{-1}\left[x_{\rho}(t)-x^{*}(t)\right]-\widehat{x}(t), & \tilde{z}_{\rho}(t)=\rho^{-1}\left[z_{\rho}(t)-z^{*}(t)\right]-\widehat{z}(t), \\
\tilde{y}_{\rho}(t)=\rho^{-1}\left[y_{\rho}(t)-y^{*}(t)\right]-\widehat{y}(t), & \tilde{q}_{\rho}(t)=\rho^{-1}\left[q_{\rho}(t)-q^{*}(t)\right]-\widehat{q}(t) .
\end{array}
$$

We have the following convergence.

Lemma 2.5. Assuming (H1) (H3) one has

$$
\begin{array}{ll}
\lim _{\rho \rightarrow 0} \sup _{0 \leq t \leq T} E\left[\left|\tilde{x}_{\rho}(t)\right|^{2}\right]=0, & \lim _{\rho \rightarrow 0} E\left[\int_{0}^{T}\left\|\tilde{z}_{\rho}(t)\right\|^{2} d t\right]=0, \\
\lim _{\rho \rightarrow 0} \sup _{0 \leq t \leq T} E\left[\left|\tilde{y}_{\rho}(t)\right|^{2}\right]=0, & \lim _{\rho \rightarrow 0} E\left[\int_{0}^{T}\left\|\tilde{q}_{\rho}(t)\right\|^{2} d t\right]=0 .
\end{array}
$$

Proof. From (2.13) and (2.20), we have

$$
\begin{aligned}
-d \tilde{y}_{\rho}(t)= & \rho^{-1}\left[f\left(x_{\rho}(t), z_{\rho}(t), y_{\rho}(t), q_{\rho}(t), u_{\rho}(t), t\right)-f\left(x^{*}(t), z^{*}(t), y^{*}(t), q^{*}(t), u^{*}(t), t\right)\right. \\
& \left.-\rho f_{x}^{*}(t) \widehat{x}(t)-\rho f_{z}^{*}(t) \widehat{z}(t)-\rho f_{y}^{*}(t) \widehat{y}(t)-\rho f_{q}^{*}(t) \widehat{q}(t)-\rho f_{u}^{*}(t)\left(u(\cdot)-u^{*}(\cdot)\right)\right] d t \\
+ & \rho^{-1}\left[g\left(x_{\rho}(t), z_{\rho}(t), y_{\rho}(t), q_{\rho}(t), u_{\rho}(t), t\right)-g\left(x^{*}(t), z^{*}(t), y^{*}(t), q^{*}(t), u^{*}(t), t\right)\right. \\
& \left.\quad-\rho g_{x}^{*}(t) \widehat{x}(t)-\rho g_{z}^{*}(t) \widehat{z}(t)-\rho g_{y}^{*}(t) \widehat{y}(t)-\rho g_{q}^{*}(t) \widehat{q}(t)-\rho g_{u}^{*}(t)\left(u(\cdot)-u^{*}(\cdot)\right)\right] d B_{t} \\
- & \tilde{q}_{\rho}(t) d W_{t},
\end{aligned}
$$

$$
\tilde{y}_{\rho}(T)=0 .
$$


Let

$$
\begin{aligned}
& A^{\rho}(t)=\int_{0}^{1} f_{x}\left(x^{*}(t)+\lambda \rho\left(\widehat{x}(t)+\tilde{x}_{\rho}(t)\right), z^{*}(t)+\lambda \rho\left(\widehat{z}(t)+\tilde{z}_{\rho}(t)\right), y^{*}(t)+\lambda \rho\left(\widehat{y}(t)+\tilde{y}_{\rho}(t)\right),\right. \\
& \left.q^{*}(t)+\lambda \rho\left(\widehat{q}(t)+\tilde{q}_{\rho}(t)\right), u^{*}(t)+\lambda \rho\left(u(t)-u^{*}(t)\right), t\right) d \lambda, \\
& B^{\rho}(t)=\int_{0}^{1} f_{z}\left(x^{*}(t)+\lambda \rho\left(\widehat{x}(t)+\tilde{x}_{\rho}(t)\right), z^{*}(t)+\lambda \rho\left(\widehat{z}(t)+\tilde{z}_{\rho}(t)\right), y^{*}(t)+\lambda \rho\left(\widehat{y}(t)+\tilde{y}_{\rho}(t)\right),\right. \\
& \left.q^{*}(t)+\lambda \rho\left(\widehat{q}(t)+\tilde{q}_{\rho}(t)\right), u^{*}(t)+\lambda \rho\left(u(t)-u^{*}(t)\right), t\right) d \lambda, \\
& C^{\rho}(t)=\int_{0}^{1} f_{y}\left(x^{*}(t)+\lambda \rho\left(\widehat{x}(t)+\tilde{x}_{\rho}(t)\right), z^{*}(t)+\lambda \rho\left(\widehat{z}(t)+\widetilde{z}_{\rho}(t)\right), y^{*}(t)+\lambda \rho\left(\widehat{y}(t)+\tilde{y}_{\rho}(t)\right),\right. \\
& \left.q^{*}(t)+\lambda \rho\left(\widehat{q}(t)+\tilde{q}_{\rho}(t)\right), u^{*}(t)+\lambda \rho\left(u(t)-u^{*}(t)\right), t\right) d \lambda, \\
& D^{\rho}(t)=\int_{0}^{1} f_{q}\left(x^{*}(t)+\lambda \rho\left(\widehat{x}(t)+\tilde{x}_{\rho}(t)\right), z^{*}(t)+\lambda \rho\left(\widehat{z}(t)+\tilde{z}_{\rho}(t)\right), y^{*}(t)+\lambda \rho\left(\widehat{y}(t)+\tilde{y}_{\rho}(t)\right),\right. \\
& \left.q^{*}(t)+\lambda \rho\left(\widehat{q}(t)+\tilde{q}_{\rho}(t)\right), u^{*}(t)+\lambda \rho\left(u(t)-u^{*}(t)\right), t\right) d \lambda, \\
& E^{\rho}(t)=\left[A^{\rho}(t)-f_{x}^{*}(t)\right] \widehat{x}(t)+\left[B^{\rho}(t)-f_{z}^{*}(t)\right] \widehat{z}(t)+\left[C^{\rho}(t)-f_{y}^{*}(t)\right] \widehat{y}(t)+\left[D^{\rho}(t)-f_{q}^{*}(t)\right] \widehat{q}(t) \\
& +\int_{0}^{1}\left[f _ { u } \left(x^{*}(t)+\lambda \rho\left(\widehat{x}(t)+\widetilde{x}_{\rho}(t)\right), z^{*}(t)+\lambda \rho\left(\widehat{z}(t)+\widetilde{z}_{\rho}(t)\right), y^{*}(t)+\lambda \rho\left(\widehat{y}(t)+\tilde{y}_{\rho}(t)\right),\right.\right. \\
& \left.\left.q^{*}(t)+\lambda \rho\left(\widehat{q}(t)+\tilde{q}_{\rho}(t)\right), u^{*}(t)+\lambda \rho\left(u(t)-u^{*}(t)\right), t\right)-f_{u}^{*}(t)\right]\left(u(t)-u^{*}(t)\right) d \lambda, \\
& A_{1}^{\rho}(t)=\int_{0}^{1} g_{x}\left(x^{*}(t)+\lambda \rho\left(\widehat{x}(t)+\tilde{x}_{\rho}(t)\right), z^{*}(t)+\lambda \rho\left(\widehat{z}(t)+\widetilde{z}_{\rho}(t)\right), y^{*}(t)+\lambda \rho\left(\widehat{y}(t)+\tilde{y}_{\rho}(t)\right),\right. \\
& \left.q^{*}(t)+\lambda \rho\left(\widehat{q}(t)+\tilde{q}_{\rho}(t)\right), u^{*}(t)+\lambda \rho\left(u(t)-u^{*}(t)\right), t\right) d \lambda, \\
& B_{1}^{\rho}(t)=\int_{0}^{1} g_{z}\left(x^{*}(t)+\lambda \rho\left(\widehat{x}(t)+\tilde{x}_{\rho}(t)\right), z^{*}(t)+\lambda \rho\left(\widehat{z}(t)+\tilde{z}_{\rho}(t)\right), y^{*}(t)+\lambda \rho\left(\widehat{y}(t)+\tilde{y}_{\rho}(t)\right),\right. \\
& \left.q^{*}(t)+\lambda \rho\left(\widehat{q}(t)+\tilde{q}_{\rho}(t)\right), u^{*}(t)+\lambda \rho\left(u(t)-u^{*}(t)\right), t\right) d \lambda, \\
& C_{1}^{\rho}(t)=\int_{0}^{1} g_{y}\left(x^{*}(t)+\lambda \rho\left(\widehat{x}(t)+\tilde{x}_{\rho}(t)\right), z^{*}(t)+\lambda \rho\left(\widehat{z}(t)+\tilde{z}_{\rho}(t)\right), y^{*}(t)+\lambda \rho\left(\widehat{y}(t)+\tilde{y}_{\rho}(t)\right),\right. \\
& \left.q^{*}(t)+\lambda \rho\left(\widehat{q}(t)+\tilde{q}_{\rho}(t)\right), u^{*}(t)+\lambda \rho\left(u(t)-u^{*}(t)\right), t\right) d \lambda, \\
& D_{1}^{\rho}(t)=\int_{0}^{1} g_{q}\left(x^{*}(t)+\lambda \rho\left(\widehat{x}(t)+\tilde{x}_{\rho}(t)\right), z^{*}(t)+\lambda \rho\left(\widehat{z}(t)+\widetilde{z}_{\rho}(t)\right), y^{*}(t)+\lambda \rho\left(\widehat{y}(t)+\tilde{y}_{\rho}(t)\right),\right. \\
& \left.q^{*}(t)+\lambda \rho\left(\widehat{q}(t)+\tilde{q}_{\rho}(t)\right), u^{*}(t)+\lambda \rho\left(u(t)-u^{*}(t)\right), t\right) d \lambda,
\end{aligned}
$$




$$
\begin{gathered}
E_{1}^{\rho}(t)=\left[A_{1}^{\rho}(t)-g_{x}^{*}(t)\right] \widehat{x}(t)+\left[B_{1}^{\rho}(t)-g_{z}^{*}(t)\right] \widehat{z}(t)+\left[C_{1}^{\rho}(t)-g_{y}^{*}(t)\right] \widehat{y}(t)+\left[D_{1}^{\rho}(t)-g_{q}^{*}(t)\right] \widehat{q}(t) \\
+\int_{0}^{1}\left[g _ { u } \left(x^{*}(t)+\lambda \rho\left(\widehat{x}(t)+\tilde{x}_{\rho}(t)\right), z^{*}(t)+\lambda \rho\left(\widehat{z}(t)+\tilde{z}_{\rho}(t)\right), y^{*}(t)+\lambda \rho\left(\widehat{y}(t)+\tilde{y}_{\rho}(t)\right),\right.\right. \\
\left.\left.q^{*}(t)+\lambda \rho\left(\widehat{q}(t)+\tilde{q}_{\rho}(t)\right), u^{*}(t)+\lambda \rho\left(u(t)-u^{*}(t)\right), t\right)-g_{u}^{*}(t)\right]\left(u(t)-u^{*}(t)\right) d \lambda .
\end{gathered}
$$

Thus

$$
\begin{gathered}
-d \tilde{y}_{\rho}(t)=\left(A^{\rho}(t) \tilde{x}_{\rho}(t)+B^{\rho}(t) \tilde{z}_{\rho}(t)+C^{\rho}(t) \tilde{y}_{\rho}(t)+D^{\rho}(t) \tilde{q}_{\rho}(t)+E^{\rho}(t)\right) d t \\
+\left(A_{1}^{\rho}(t) \tilde{x}_{\rho}(t)+B_{1}^{\rho}(t) \tilde{z}_{\rho}(t)+C_{1}^{\rho}(t) \tilde{y}_{\rho}(t)+D_{1}^{\rho}(t) \tilde{q}_{\rho}(t)+E_{1}^{\rho}(t)\right) d B_{t}-\tilde{q}_{\rho}(t) d W(t), \\
\tilde{y}_{\rho}(T)=0 .
\end{gathered}
$$

Using Lemma 2.1 to $\left|\tilde{y}_{\rho}(t)\right|^{2}$, we get

$$
\begin{aligned}
& E\left|\tilde{y}_{\rho}(t)\right|^{2}+E \int_{t}^{T}\left\|\tilde{q}_{\rho}(s)\right\|^{2} d s \\
&=2 E \int_{t}^{T}\left(\tilde{y}_{\rho}(t), A^{\rho}(s) \tilde{x}_{\rho}(s)+B^{\rho}(s) \tilde{z}_{\rho}(s)+C^{\rho}(s) \tilde{y}_{\rho}(s)+D^{\rho}(s) \tilde{q}_{\rho}(s)+E^{\rho}(s)\right) d s \\
&+E \int_{t}^{T}\left\|A_{1}^{\rho}(s) \tilde{x}_{\rho}(s)+B_{1}^{\rho}(s) \tilde{z}_{\rho}(s)+C_{1}^{\rho}(s) \tilde{y}_{\rho}(s)+D_{1}^{\rho}(s) \tilde{q}_{\rho}(s)+E_{1}^{\rho}(s)\right\|^{2} d s \\
& \leq K\left(E \int_{t}^{T}\left|\tilde{y}_{\rho}(s)\right|^{2} d s+E \int_{t}^{T}\left|\tilde{x}_{\rho}(s)\right|^{2} d s\right) \\
&+\beta_{1}\left(E \int_{t}^{T}\left\|\tilde{z}_{\rho}(s)\right\|^{2} d s+E \int_{t}^{T}\left\|\tilde{q}_{\rho}(s)\right\|^{2} d s+J_{\rho}\right),
\end{aligned}
$$

where $K>0,0<\beta_{1}<1 / 2$ are constants and $J_{\rho}=E \int_{t}^{T}\left|E^{\rho}(s)\right|^{2} d s+E \int_{t}^{T}\left|E_{1}^{\rho}(s)\right|^{2} d s$.

Similar analysis shows that

$$
\begin{aligned}
-d \tilde{x}_{\rho}(t)= & \left(A^{\prime} \rho(t) \tilde{x}_{\rho}(t)+B^{\prime} \rho(t) \tilde{z}_{\rho}(t)+C^{\prime} \rho(t) \tilde{y}_{\rho}(t)+D^{\prime \rho}(t) \tilde{q}_{\rho}(t)+E^{\prime} \rho(t)\right) d t \\
& +\left(A_{1}^{\prime}(t) \tilde{x}_{\rho}(t)+B_{1}^{\prime}(t) \tilde{z}_{\rho}(t)+C_{1}^{\prime}(t) \tilde{y}_{\rho}(t)+D_{1}^{\prime \rho}(t) \tilde{q}_{\rho}(t)+E_{1}^{\prime}(t)\right) d W_{t}-\tilde{z}_{\rho}(t) d B(t), \\
& \tilde{x}_{\rho}(0)=0,
\end{aligned}
$$

where $H^{\prime} \rho(t)$ and $H_{1}^{\prime}(t)(H=A, B, C, D$ and $E)$ are similarly defined as before. 
It yields that

$$
\begin{aligned}
& E\left|\tilde{x}_{\rho}(t)\right|^{2}+E \int_{t}^{T}\left\|\tilde{z}_{\rho}(s)\right\|^{2} d s \\
& \quad=2 E \int_{t}^{T}\left(\tilde{x}_{\rho}(t), A^{\prime} \rho(s) \tilde{x}_{\rho}(s)+B^{\prime} \rho(s) \tilde{z}_{\rho}(s)+C^{\prime} \rho(s) \tilde{y}_{\rho}(s)+D^{\prime} \rho(s) \tilde{q}_{\rho}(t s)+E^{\prime} \rho(s)\right) d s \\
& \quad+E \int_{t}^{T}\left\|A_{1}^{\prime \rho}(s) \tilde{x}_{\rho}(s)+B_{1}^{\prime}(s) \tilde{z}_{\rho}(s)+C_{1}^{\prime}(s) \tilde{y}_{\rho}(s)+D_{1}^{\prime \rho}(s) \tilde{q}_{\rho}(s)+E_{1}^{\prime}(s)\right\|^{2} d s \\
& \leq K E\left[\int_{t}^{T}\left|\tilde{y}_{\rho}(s)\right|^{2} d s+\int_{t}^{T}\left|\tilde{x}_{\rho}(s)\right|^{2} d s\right]+\beta_{2} E\left[\int_{t}^{T}\left\|\tilde{z}_{\rho}(s)\right\|^{2} d s+\int_{t}^{T}\left\|\tilde{q}_{\rho}(s)\right\|^{2} d s+J_{\rho}^{\prime}\right],
\end{aligned}
$$

where $K>0,0<\beta_{2}<1 / 2$ are constants and $J_{\rho}^{\prime}=E\left[\int_{t}^{T}\left|E^{\prime} \rho(s)\right|^{2} d s+\int_{t}^{T}\left|E_{1}^{\prime \rho}(s)\right|^{2} d s\right]$. Since $0<\beta_{1}+\beta_{2}<1$ and $K>0$, there exists $K_{1}>0$ such that

$$
\begin{gathered}
E\left|\tilde{x}_{\rho}(t)\right|^{2}+E\left|\tilde{y}_{\rho}(t)\right|^{2}+\left(1-\beta_{1}-\beta_{2}\right)\left(E \int_{t}^{T}\left\|\tilde{z}_{\rho}(s)\right\|^{2} d s+E \int_{t}^{T}\left\|\tilde{q}_{\rho}(s)\right\|^{2} d s\right) \\
\leq K_{1}\left(E \int_{t}^{T}\left|\tilde{x}_{\rho}(s)\right|^{2} d s+E \int_{t}^{T}\left|\tilde{y}_{\rho}(s)\right|^{2} d s+J_{\rho}+J_{\rho}^{\prime}\right) .
\end{gathered}
$$

Since the Lebesgue dominated convergence theorem implies $\lim _{\rho \rightarrow 0} J_{\rho}=0, \lim _{\rho \rightarrow 0} J_{\rho}^{\prime}=0$, we obtain the result by Gronwall's inequality.

\subsubsection{Variational Inequality}

In this subsection, we apply Ekeland's variational principle [28] to deal with initial-terminal state constraints:

$$
E\left(\psi\left(x_{T}^{(\xi, \eta, u(\cdot))}\right)\right)=a, \quad E\left(h\left(y_{0}^{(\xi, \eta, u(\cdot))}\right)\right)=b
$$

Define

$$
\begin{aligned}
F_{\varepsilon}((\xi, \eta, u(\cdot))) \triangleq & \left\{\left|E\left(\psi\left(x_{T}^{(\xi, \eta, u(\cdot))}\right)\right)-a\right|^{2}+\left|E\left(h\left(y_{0}^{(\xi, \eta, u(\cdot))}\right)\right)-b\right|^{2}\right. \\
& +\left(\max \left(0, E\left[\phi\left(x^{*}(T)\right)-\phi\left(x_{T}^{(\xi, \eta, u(\cdot))}\right)\right]+\varepsilon\right)\right)^{2} \\
& \left.+\left(\max \left(0, E\left[\gamma\left(y^{*}(0)\right)-\gamma\left(y_{0}^{(\xi, \eta, u(\cdot))}\right)\right]+\varepsilon\right)\right)^{2}\right\}^{1 / 2}
\end{aligned}
$$

where $a$ and $b$ are the given initial and terminal state constraints and $\varepsilon$ is an arbitrary positive constant. 
It is easy to check that the mappings $\left|E\left(\psi\left(x_{T}^{(\xi, \eta, u(\cdot))}\right)\right)-a\right|^{2},\left|E\left(h\left(y_{0}^{(\xi, \eta, u(\cdot))}\right)\right)-b\right|^{2}$, $\phi\left(x_{T}^{(\xi, \eta, u(\cdot))}\right)$ and $\gamma\left(y_{0}^{(\xi, \eta, u(\cdot))}\right)$ are all continuous functionals from $U$ to $\mathbb{R}$.

Theorem 2.6. Suppose (H1) (H3). Let $\left(\xi^{*}, \eta^{*}, u^{*}(\cdot)\right)$ be an optimal solution to (2.17). Then there exist $h_{0}, h_{1}, h_{2}, h_{3} \in \mathbb{R}$ with $h_{0}, h_{1} \leq 0$ and $\left|h_{0}\right|+\left|h_{1}\right|+\left|h_{2}\right|+\left|h_{3}\right| \neq 0$ such that the following variational inequality holds:

$$
\begin{aligned}
& h_{3} E\left\langle\psi_{x}\left(x^{*}(T)\right), \widehat{x}(T)\right\rangle+h_{2} E\left\langle h_{y}\left(y^{*}(0)\right), \widehat{y}(0)\right\rangle+h_{1} E\left\langle\phi_{x}\left(x^{*}(T)\right), \widehat{x}(T)\right\rangle \\
& \quad+h_{0} E\left\langle\gamma_{y}\left(y^{*}(0)\right), \widehat{y}(0)\right\rangle \geq 0,
\end{aligned}
$$

where $\widehat{x}(T)$ is the solution $\widehat{x}(\cdot)$ of (2.20) at time $T$ and $\widehat{y}(0)$ is the solution $\widehat{y}(\cdot)$ of (2.20) at time 0 .

Proof. It is easy to check that $F_{\varepsilon}(\cdot)$ is continuous on $U$ such that

$$
\begin{gathered}
F_{\varepsilon}\left(\xi^{*}, \eta^{*}, u^{*}(\cdot)\right)=\sqrt{2} \varepsilon, \\
F_{\varepsilon}(\xi, \eta, u(\cdot))>0, \quad \forall(\xi, \eta, u(\cdot)) \in U, \\
F_{\varepsilon}\left(\xi^{*}, \eta^{*}, u^{*}(\cdot)\right) \leq \inf _{(\xi, \eta, u(\cdot)) \in U} F_{\varepsilon}(\xi, \eta, u(\cdot))+\sqrt{2} \varepsilon .
\end{gathered}
$$

Thus, from Ekeland's variational principle [28], $\exists\left(\xi^{\varepsilon}, \eta^{\varepsilon}, u^{\varepsilon}(\cdot)\right) \in U$ such that

(i) $F_{\varepsilon}\left(\xi^{\varepsilon}, \eta^{\varepsilon}, u^{\varepsilon}(\cdot)\right) \leq F_{\varepsilon}\left(\xi^{*}, \eta^{*}, u^{*}(\cdot)\right)$,

(ii) $d\left(\left(\xi^{*}, \eta^{*}, u^{*}(\cdot)\right),\left(\xi^{\varepsilon}, \eta^{\varepsilon}, u^{\varepsilon}(\cdot)\right)\right) \leq \sqrt{2 \varepsilon}$,

(iii) $F_{\varepsilon}(\xi, \eta, u(\cdot))+\sqrt{2 \varepsilon} d\left((\xi, \eta, u(\cdot)),\left(\xi^{\varepsilon}, \eta^{\varepsilon}, u^{\varepsilon}(\cdot)\right)\right) \geq F_{\varepsilon}\left(\xi^{\varepsilon}, \eta^{\varepsilon}, u^{\varepsilon}(\cdot)\right)$, for all $(\xi, \eta, u(\cdot)) \in$ $U$.

For any $(\xi, \eta, u(\cdot)) \in U$ and $0 \leq \rho \leq 1$, set $\left(\xi_{\rho}^{\varepsilon}, \eta_{\rho}^{\varepsilon}, u_{\rho}^{\varepsilon}(\cdot)\right)=\left(\xi^{\varepsilon}+\rho\left(\xi-\xi^{\varepsilon}\right), \eta^{\varepsilon}+\rho\left(\eta-\eta^{\varepsilon}\right), u^{\varepsilon}(\cdot)+\right.$ $\left.\rho\left(u(\cdot)-u^{\varepsilon}(\cdot)\right)\right)$. Let $\left(x_{\rho}^{\varepsilon}(\cdot), y_{\rho}^{\varepsilon}(\cdot), z_{\rho}^{\varepsilon}(\cdot), q_{\rho}^{\varepsilon}(\cdot)\right)\left(\operatorname{resp} .,\left(x^{\varepsilon}(\cdot), y^{\varepsilon}(\cdot), z^{\varepsilon}(\cdot), q^{\varepsilon}(\cdot)\right)\right)$ be the solution of (2.13) under $\left(\xi_{\rho}^{\varepsilon}, \eta_{\rho}^{\varepsilon}, u_{\rho}^{\varepsilon}(\cdot)\right)$ (resp., $\left(\xi^{\varepsilon}, \eta^{\varepsilon}, u^{\varepsilon}(\cdot)\right)$, and let $\left(\widehat{x}^{\varepsilon}(\cdot), \widehat{y}^{\varepsilon}(\cdot), \widehat{z}^{\varepsilon}(\cdot), \widehat{q}^{\varepsilon}(\cdot)\right)$ be the solution of $(2.20)$ in which $\left(\xi^{*}, \eta^{*}, u^{*}(\cdot)\right)$ is substituted by $\left(\xi^{\varepsilon}, \eta^{\varepsilon}, u^{\varepsilon}(\cdot)\right)$.

From (iii), we know that

$$
F_{\varepsilon}\left(\xi_{\rho}^{\varepsilon}, \eta_{\rho}^{\varepsilon}, u_{\rho}^{\varepsilon}(\cdot)\right)-F_{\varepsilon}\left(\xi^{\varepsilon}, \eta^{\varepsilon}, u^{\varepsilon}(\cdot)\right)+\sqrt{2 \varepsilon} d\left(\left(\xi_{\rho}^{\varepsilon}, \eta_{\rho}^{\varepsilon}, u_{\rho}^{\varepsilon}(\cdot)\right),\left(\xi^{\varepsilon}, \eta^{\varepsilon}, u^{\varepsilon}(\cdot)\right)\right) \geq 0
$$

On the other hand, similarly to Lemma 2.5 we have

$$
\begin{aligned}
& \lim _{\rho \rightarrow 0} \sup _{0 \leq t \leq T} E\left[\rho^{-1}\left[x_{\rho}^{\varepsilon}(t)-x^{\varepsilon}(t)\right]-\widehat{x}^{\varepsilon}(t)\right]^{2}=0 \\
& \lim _{\rho \rightarrow 0} \sup _{0 \leq t \leq T} E\left[\rho^{-1}\left[y_{\rho}^{\varepsilon}(t)-y^{\varepsilon}(t)\right]-\widehat{y}^{\varepsilon}(t)\right]^{2}=0 .
\end{aligned}
$$


This leads to the following expansions:

$$
\begin{aligned}
& \mid E\left(\phi\left(x_{\rho}^{\varepsilon}(T)\right)-\left.a\right|^{2}-\mid E\left(\phi\left(x^{\varepsilon}(T)\right)-\left.a\right|^{2}=2 \rho\left\langle E \phi\left(x^{\varepsilon}(T)\right)-a, E\left[\phi_{x}\left(x^{\varepsilon}(T)\right) \widehat{x}^{\varepsilon}(T)\right]\right\rangle+o(\rho),\right.\right. \\
& \mid E\left(h\left(y_{\rho}^{\varepsilon}(0)\right)-\left.b\right|^{2}-\mid E\left(h(y \varepsilon(0))-\left.b\right|^{2}=2 \rho\left\langle E h\left(y^{\varepsilon}(0)\right)-b, E\left[h_{y}\left(y^{\varepsilon}(0)\right) \hat{y}^{\varepsilon}(0)\right]\right\rangle+o(\rho) .\right.\right.
\end{aligned}
$$

Applying the linearization technique, then

$$
\begin{aligned}
& E\left[\phi\left(x_{\rho}^{\varepsilon}(T)\right)-\phi\left(x^{\varepsilon}(T)\right)\right]=E\left[\int_{0}^{T} \phi_{x}\left(x^{\left(\xi^{\varepsilon}+\lambda \rho \widehat{\xi}^{\varepsilon}, \eta^{\varepsilon}+\lambda \rho \widehat{\eta}^{\varepsilon}, u^{\varepsilon}(\cdot)+\lambda \rho \widehat{u}^{\varepsilon}\right)}(T)\right) d \lambda \cdot \rho \widehat{x}^{\varepsilon}(T)\right] \\
& E\left[\gamma\left(y_{\rho}^{\varepsilon}(0)\right)-\gamma\left(y^{\varepsilon}(0)\right)\right]=E\left[\int_{0}^{T} r_{y}\left(y^{\left(\xi^{\varepsilon}+\lambda \rho \hat{\xi}^{\varepsilon}, \eta^{\varepsilon}+\lambda \rho \hat{\eta}^{\varepsilon}, u^{\varepsilon}(\cdot)+\lambda \rho \hat{u}^{\varepsilon}(\cdot)\right)}(0)\right) d \lambda \cdot \rho \widehat{y}^{\varepsilon}(0)\right] .
\end{aligned}
$$

So we have the following expansions:

$$
\begin{gathered}
\left(E\left[\phi\left(x^{*}(T)\right)-\phi\left(x_{\rho}^{\varepsilon}(T)\right]+\varepsilon\right)^{2}-\left(E\left[\phi\left(x^{*}(T)\right)-\phi\left(x^{\varepsilon}(T)\right]+\varepsilon\right)^{2}\right.\right. \\
=-2 \rho E\left[\phi_{x}\left(x^{\varepsilon}(T)\right) \cdot \rho \widehat{x}_{T}^{\varepsilon}\right]\left[E\left[\phi\left(x^{*}(T)\right)-\phi\left(x^{\varepsilon}(T)\right]+\varepsilon\right],\right. \\
\left(E \left[\gamma\left(y^{*}(0)-\gamma\left(y_{\rho}^{\varepsilon}(0)\right]+\varepsilon\right)^{2}-\left(E\left[\gamma\left(y^{*}(0)\right)-\gamma\left(y^{\varepsilon}(0)\right)\right]+\varepsilon\right)^{2}\right.\right. \\
=-2 \rho E\left[\gamma_{y}\left(y^{\varepsilon}(0)\right) \cdot \rho \widehat{y}_{0}^{\varepsilon}\right]\left[E\left[\gamma\left(y^{*}(0)\right)-\gamma\left(y^{\varepsilon}(0)\right)\right]+\varepsilon\right] .
\end{gathered}
$$

For the given $\varepsilon$, we consider the following four cases.

Case 1 . There exists $\rho_{0}>0$ such that

$$
E\left[\phi\left(x_{\rho}^{\varepsilon}(T)\right)-\phi\left(x^{\varepsilon}(T)\right)\right]+\varepsilon>0, \quad E\left[\gamma\left(y_{\rho}^{\varepsilon}(0)\right)-\gamma\left(y^{\varepsilon}(0)\right)\right]+\varepsilon>0
$$

for all $\rho \in\left(0, \rho_{0}\right)$. 
In this case,

$$
\begin{aligned}
& \lim _{\rho \rightarrow 0} \frac{F_{\varepsilon}\left(\xi_{\rho}^{\varepsilon}, \eta_{\rho}^{\varepsilon}, u_{\rho}^{\varepsilon}(\cdot)\right)-F_{\varepsilon}\left(\xi^{\varepsilon}, \eta^{\varepsilon}, u^{\varepsilon}(\cdot)\right)}{\rho} \\
&=\lim _{\rho \rightarrow 0} \frac{1}{F_{\varepsilon}\left(\xi_{\rho}^{\varepsilon}, \eta_{\rho}^{\varepsilon}, u_{\rho}^{\varepsilon}(\cdot)\right)+F_{\varepsilon}\left(\xi^{\varepsilon}, \eta^{\varepsilon}, u^{\varepsilon}(\cdot)\right)} \frac{F_{\varepsilon}^{2}\left(\xi_{\rho}^{\varepsilon}, \eta_{\rho}^{\varepsilon}, u_{\rho}^{\varepsilon}(\cdot)\right)-F_{\varepsilon}^{2}\left(\xi^{\varepsilon}, \eta^{\varepsilon}, u^{\varepsilon}(\cdot)\right)}{\rho} \\
&=\frac{1}{F_{\varepsilon}\left(\xi^{\varepsilon}, \eta^{\varepsilon}, u^{\varepsilon}(\cdot)\right)}\left\{\left\langle E \psi\left(x^{\varepsilon}(T)\right)-a, E\left[\psi_{x}\left(x^{\varepsilon}(T)\right) \widehat{x}^{\varepsilon}(T)\right]\right\rangle\right. \\
&+\left\langle E h\left(y^{\varepsilon}(0)\right)-b, E\left[h_{y}\left(y^{\varepsilon}(0)\right) \widehat{y}^{\varepsilon}(0)\right]\right\rangle \\
&-\left\langle E\left[\phi\left(x^{*}(T)\right)-\phi\left(x^{\varepsilon}(T)\right)\right]+\varepsilon, E\left[\phi_{x}\left(x^{\varepsilon}(T)\right) \widehat{x}^{\varepsilon}(T)\right]\right\rangle \\
&\left.-\left\langle E\left[\gamma\left(y^{*}(0)\right)-\gamma\left(y^{\varepsilon}(0)\right)\right]+\varepsilon, E\left[\gamma_{y}\left(y^{\varepsilon}(0)\right) \hat{y}^{\varepsilon}(0)\right]\right\rangle\right\} .
\end{aligned}
$$

Dividing (2.34) by $\rho$ and sending $\rho$ to 0 , we obtain

$$
\begin{aligned}
& h_{3}^{\varepsilon} E\left\langle\psi_{x}\left(x^{\varepsilon}(T)\right), \widehat{x}^{\varepsilon}(T)\right\rangle+h_{2}^{\varepsilon} E\left\langle h_{y}\left(y^{\varepsilon}(0)\right), \widehat{y}^{\varepsilon}(0)\right\rangle \\
& \quad+h_{1}^{\varepsilon} E\left\langle\phi_{x}\left(x^{\varepsilon}(T)\right), \widehat{x}^{\varepsilon}(T)\right\rangle+h_{0}^{\varepsilon} E\left\langle\gamma_{y}\left(y^{\varepsilon}(0), \widehat{y}^{\varepsilon}(0)\right\rangle \geq-\sqrt{2 \varepsilon} d\left(\left(\xi^{\varepsilon}, \eta^{\varepsilon}, u^{\varepsilon}(\cdot)\right),(\xi, \eta, u(\cdot))\right),\right.
\end{aligned}
$$

where

$$
\begin{gathered}
h_{0}^{\varepsilon}=-\frac{1}{F_{\varepsilon}\left(\xi^{\varepsilon}, \eta^{\varepsilon}, u^{\varepsilon}(\cdot)\right)}\left[E\left[\gamma\left(y^{*}(0)\right)-\gamma\left(y^{\varepsilon}(0)\right)\right]+\varepsilon\right] \leq 0, \\
h_{1}^{\varepsilon}=-\frac{1}{F_{\varepsilon}\left(\xi^{\varepsilon}, \eta^{\varepsilon}, u^{\varepsilon}(\cdot)\right)}\left[E\left[\phi\left(x^{*}(T)\right)-\phi\left(x^{\varepsilon}(T)\right)\right]+\varepsilon\right] \leq 0, \\
h_{2}^{\varepsilon}=\frac{1}{F_{\varepsilon}\left(\xi^{\varepsilon}, \eta^{\varepsilon}, u^{\varepsilon}(\cdot)\right)}\left[E h\left(y^{\varepsilon}(0)\right)-b\right], \\
h_{3}^{\varepsilon}=\frac{1}{F_{\varepsilon}\left(\xi^{\varepsilon}, \eta^{\varepsilon}, u^{\varepsilon}(\cdot)\right)}\left[E \psi\left(x^{\varepsilon}(T)\right)-a\right] .
\end{gathered}
$$

Case 2. There exists a position sequence $\left\{\rho_{n}\right\}$ satisfying $\rho_{n} \rightarrow 0$ such that

$$
E\left[\phi\left(x_{\rho_{n}}^{\varepsilon}(T)\right)-\phi\left(x^{*}(T)\right)\right]+\varepsilon \leq 0, \quad E\left[\gamma\left(y_{\rho_{n}}^{\varepsilon}(0)\right)-\gamma\left(y^{*}(0)\right)\right]+\varepsilon>0 .
$$


Then

$$
\begin{aligned}
F_{\varepsilon}\left(\xi_{\rho_{n}}^{\varepsilon}, \eta_{\rho_{n}}^{\varepsilon}, u_{\rho_{n}}^{\varepsilon}(\cdot)\right)=\left\{\left|E \psi\left(x_{\rho_{n}}^{\varepsilon}(T)\right)-a\right|^{2}+\left|E h\left(y_{\rho_{n}}^{\varepsilon}(0)\right)-b\right|^{2}\right. \\
\left.+\left(\max \left(0, E\left[\gamma\left(y^{*}(0)\right)-\gamma\left(y_{\rho_{n}}^{\varepsilon}(0)\right)\right]+\varepsilon\right)\right)^{2}\right\}^{1 / 2}
\end{aligned}
$$

For sufficiently large $n$, since $F_{\varepsilon}(\cdot)$ is continuous, we conclude

$$
\begin{aligned}
F_{\varepsilon}\left(\xi^{\varepsilon}, \eta^{\varepsilon}, u^{\varepsilon}(\cdot)\right)= & \left\{\left|E \psi\left(x^{\varepsilon}(T)\right)-a\right|^{2}\right\}^{1 / 2}+\left|E h\left(y^{\varepsilon}(0)\right)-b\right|^{2} \\
& \left.+\left(\max \left(0, E\left[\gamma\left(y^{*}(0)\right)-\gamma\left(y^{\varepsilon}(0)\right)\right]+\varepsilon\right)\right)^{2}\right\}^{1 / 2}
\end{aligned}
$$

Now

$$
\begin{aligned}
& \lim _{n \rightarrow \infty} \frac{F_{\varepsilon}\left(\xi_{\rho_{n}}^{\varepsilon}, \eta_{\rho_{n}}^{\varepsilon}, u_{\rho_{n}}^{\varepsilon}(\cdot)\right)-F_{\varepsilon}\left(\xi^{\varepsilon}, \eta^{\varepsilon}, u^{\varepsilon}(\cdot)\right)}{\rho_{n}} \\
&=\lim _{n \rightarrow \infty} \frac{1}{F_{\varepsilon}\left(\xi_{\rho_{n}}^{\varepsilon}, \eta_{\rho_{n}}^{\varepsilon}, u_{\rho_{n}}^{\varepsilon}(\cdot)\right)+F_{\varepsilon}\left(\xi^{\varepsilon}, \eta^{\varepsilon}, u^{\varepsilon}(\cdot)\right)} \frac{F_{\varepsilon}^{2}\left(\xi_{\rho_{n}}^{\varepsilon}, \eta_{\rho_{n}}^{\varepsilon}, u_{\rho_{n}}^{\varepsilon}(\cdot)\right)-F_{\varepsilon}^{2}\left(\xi^{\varepsilon}, \eta^{\varepsilon}, u^{\varepsilon}(\cdot)\right)}{\rho_{n}} \\
&=\frac{1}{F_{\varepsilon}\left(\xi^{\varepsilon}, \eta^{\varepsilon}, u^{\varepsilon}(\cdot)\right)}\left\{\left\langle E \psi\left(x^{\varepsilon}(T)\right)-a, E\left[\psi_{x}\left(x^{\varepsilon}(T)\right) \widehat{x}^{\varepsilon}(T)\right]\right\rangle\right. \\
&+\left\langle E h\left(y^{\varepsilon}(0)\right)-b, E\left[h_{y}\left(y^{\varepsilon}(0)\right) \widehat{y}^{\varepsilon}(0)\right]\right\rangle \\
&\left.-\left\langle E\left[\gamma\left(y^{*}(0)\right)-\gamma\left(y^{\varepsilon}(0)\right)\right]+\varepsilon, E\left[\gamma_{y}\left(y^{\varepsilon}(0)\right) \widehat{y}^{\varepsilon}(0)\right]\right\rangle\right\} .
\end{aligned}
$$

Similar to Case 1 we get

$$
\begin{gathered}
h_{3}^{\varepsilon} E\left\langle\psi_{x}\left(x^{\varepsilon}(T)\right), \widehat{x}^{\varepsilon}(T)\right\rangle+h_{2}^{\varepsilon} E\left\langle h_{y}\left(y^{\varepsilon}(0)\right), \widehat{y}^{\varepsilon}(0)\right\rangle+h_{0}^{\varepsilon} E\left\langle\gamma_{y}\left(y^{\varepsilon}(0)\right), \widehat{y}^{\varepsilon}(0)\right\rangle \\
\geq-\sqrt{2 \varepsilon} d\left(\left(\xi^{\varepsilon}, \eta^{\varepsilon}, u^{\varepsilon}(\cdot)\right),(\xi, \eta, u(\cdot))\right)
\end{gathered}
$$

where

$$
\begin{gathered}
h_{0}^{\varepsilon}=-\frac{1}{F_{\varepsilon}\left(\xi^{\varepsilon}, \eta^{\varepsilon}, u^{\varepsilon}(\cdot)\right)}\left[E\left[\gamma\left(y^{*}(0)\right)-\gamma\left(y^{\varepsilon}(0)\right)\right]+\varepsilon\right] \leq 0, \\
h_{1}^{\varepsilon}=0, \\
h_{2}^{\varepsilon}=\frac{1}{F_{\varepsilon}\left(\xi^{\varepsilon}, \eta^{\varepsilon}, u^{\varepsilon}(\cdot)\right)}\left[E h\left(y^{\varepsilon}(0)\right)-b\right], \\
h_{3}^{\varepsilon}=\frac{1}{F_{\varepsilon}\left(\xi^{\varepsilon}, \eta^{\varepsilon}, u^{\varepsilon}(\cdot)\right)}\left[E \psi\left(x^{\varepsilon}(T)\right)-a\right] .
\end{gathered}
$$


Case 3. There exists a positive sequence $\left\{\rho_{n}\right\}$ satisfying $\rho_{n} \rightarrow 0$ such that

$$
E\left[\phi\left(x_{\rho_{n}}^{\varepsilon}(T)\right)-\phi\left(x^{*}(T)\right)\right]+\varepsilon>0, \quad E\left[\gamma\left(y_{\rho_{n}}^{\varepsilon}(0)\right)-\gamma\left(y^{*}(0)\right)\right]+\varepsilon \leq 0
$$

Case 4 . There exists a positive sequence $\left\{\rho_{n}\right\}$ satisfying $\rho_{n} \rightarrow 0$ such that

$$
E\left[\phi\left(x_{\rho_{n}}^{\varepsilon}(T)\right)-\phi\left(x^{*}(T)\right)\right]+\varepsilon \leq 0, \quad E\left[\gamma\left(y_{\rho_{n}}^{\varepsilon}(0)\right)-\gamma\left(y^{*}(0)\right)\right]+\varepsilon \leq 0
$$

Similar techniques can be used for both Case 3 and Case 4 .

In summary, for all those cases, we have $h_{0}^{\varepsilon} \leq 0, h_{1}^{\varepsilon} \leq 0$ and $\left|h_{0}^{\varepsilon}\right|^{2}+\left|h_{1}^{\varepsilon}\right|^{2}+\left|h_{2}^{\varepsilon}\right|^{2}+\left|h_{3}^{\varepsilon}\right|^{2}=1$ by the definition of $F_{\varepsilon}(\cdot)$. Then there exists a convergent subsequence of $\left(h_{3}^{\varepsilon}, h_{2}^{\varepsilon}, h_{1}^{\varepsilon}, h_{0}^{\varepsilon}\right)$ whose limit is denoted by $\left(h_{3}, h_{2}, h_{1}, h_{0}\right)$. On the other hand, it is easy to check that $\hat{x}^{\varepsilon}(T) \rightarrow 0$, $\widehat{y}^{\varepsilon}(0) \rightarrow 0$ as $\varepsilon \rightarrow 0$. Thus (2.32) holds.

\subsubsection{Maximum Principle}

In this subsection we derive the maximum principle for the case where $l(x, z, y, q, u, t)=$ $0, x(x)=0, \lambda(y)=0$ and then present the results for the general case in Section 2.3.4. To this end, we introduce the adjoint process $(m(\cdot), p(\cdot))$, and $(n(\cdot), \delta(\cdot))$ associated with the optimal solution $\left(\xi^{*}, \eta^{*}, u^{*}(\cdot)\right)$ to $(2.13)$, which is the solution of the following time-symmetric FBDSDE:

$$
\begin{gathered}
-d m(t)=-\left(F_{x}^{*}(t) m(t)+G_{x}^{*}(t) p(t)+f_{x}^{*}(t) n(t)+g_{x}^{*}(t) \delta(t)\right) d t \\
+\left(F_{z}^{*}(t) m(t)+G_{z}^{*}(t) p(t)+f_{z}^{*}(t) n(t)+g_{z}^{*}(t) \delta(t)\right) d B_{t}-p_{t} d W_{t}, \\
m(T)=-\left(h_{3} \psi_{x}\left(x_{T}^{*}\right)+h_{1} \phi_{x}\left(x_{T}^{*}\right)\right), \\
d n(t)=\left(F_{y}^{*}(t) m(t)+G_{y}^{*}(t) p(t)+f_{y}^{*}(t) n(t)+g_{y}^{*}(t) \delta(t)\right) d t \\
+\left(F_{q}^{*}(t) m(t)+G_{q}^{*}(t) p(t)+f_{q}^{*}(t) n(t)+g_{q}^{*}(t) \delta(t)\right) d W_{t}-\delta_{t} d B_{t}, \\
n(0)=h_{2} h_{y}\left(y_{0}^{*}\right)+h_{0} \gamma_{y}\left(y_{0}^{*}\right),
\end{gathered}
$$

where $F_{k}^{*}(t), f_{k}^{*}(t), G_{k}^{*}(t), g_{k}^{*}(t)$ for $k=x, y, z, q$ are defined as in (2.20). It is easy to check that there exist unique processes $(m(\cdot), p(\cdot)),(n(\cdot), \delta(\cdot))$ which solve the above equations.

Theorem 2.7. We assume (H1) (H4). Let $\left(\xi^{*}, \eta^{*}, u^{*}(\cdot)\right)$ be optimal and let $\left(x^{*}(\cdot), z^{*}(\cdot), y^{*}(\cdot), q^{*}(\cdot)\right)$ be the corresponding optimal trajectory. Then for arbitrary $(\xi, \eta, u(\cdot)) \in U$ one has for any $t \in[0, T]$,

$$
\begin{gathered}
\left\langle m(0), \xi-\xi^{*}\right\rangle \leq 0, \\
\left\langle n(T), \eta-\eta^{*}\right\rangle \geq 0, \\
\left\langle m(t) F_{u}^{*}(t)+p(t) G_{u}^{*}(t)+n(t) f_{u}^{*}(t)+g_{u}^{*}(t) \delta(t), u(t)-u^{*}(t)\right\rangle \geq 0 .
\end{gathered}
$$


Proof. For any $(\xi, \eta, u(\cdot)) \in U$, let $(\widehat{x}(\cdot), \widehat{z}(\cdot), \widehat{y}(\cdot), \widehat{q}(\cdot))$ be the solution to (2.20). Applying Lemma 2.1 to $\langle m(t), \widehat{x}(t)\rangle+\langle n(t), \widehat{y}(t)\rangle$, we have

$$
\begin{aligned}
E\langle m(T), \widehat{x}(T)\rangle+E\langle n(T), \widehat{y}(T)\rangle-E\langle m(0), \widehat{x}(0)\rangle-E\langle n(0), \widehat{y}(0)\rangle \\
=E \int_{0}^{T} m_{t}\left(-F_{x}^{*}(t) \widehat{x}(t)-F_{z}^{*}(t) \widehat{z}(t)-F_{y}^{*}(t) \widehat{y}(t)-F_{q}^{*}(t) \widehat{q}(t)-F_{u}^{*}(t) \widehat{u}(t)\right) d t \\
\quad+E \int_{0}^{T} \widehat{x}(t)\left(F_{x}^{*}(t) m(t)+G_{x}^{*}(t) p(t)+f_{x}^{*}(t) n(t)+g_{x}^{*}(t) \delta(t)\right) d t \\
\quad+E \int_{0}^{T} \widehat{z}(t)\left(F_{z}^{*}(t) m(t)+G_{z}^{*}(t) p(t)+f_{z}^{*}(t) n(t)+g_{z}^{*}(t) \delta(t)\right) d t \\
\quad-E \int_{0}^{T} p(t)\left(G_{x}^{*}(t) \widehat{x}(t)+G_{z}^{*}(t) \widehat{z}(t)+G_{y}^{*}(t) \widehat{y}(t)+G_{q}^{*}(t) \widehat{q}(t)+G_{u}^{*}(t) \widehat{u}(t)\right) d t \\
\quad-E \int_{0}^{T} n(t)\left(f_{x}^{*}(t) \widehat{x}(t)+f_{z}^{*}(t) \widehat{z}(t)+f_{y}^{*}(t) \widehat{y}(t)+f_{q}^{*}(t) \widehat{q}(t)+f_{u}^{*}(t) \widehat{u}(t)\right) d t \\
\quad+E \int_{0}^{T} \widehat{y}(t)\left(F_{y}^{*}(t) m(t)+G_{y}^{*}(t) p(t)+f_{y}^{*}(t) n(t)+g_{y}^{*}(t) \delta(t)\right) d t \\
\quad+E \int_{0}^{T} \widehat{q}(t)\left(F_{q}^{*}(t) m(t)+G_{q}^{*}(t) p(t)+f_{q}^{*}(t) n(t)+q_{q}^{*}(t) \delta(t)\right) d t \\
\quad-E \int_{0}^{T} \delta(t)\left(g_{x}^{*}(t) \widehat{x}(t)+g_{z}^{*}(t) \widehat{z}(t)+g_{y}^{*}(t) \widehat{y}(t)+g_{q}^{*}(t) \widehat{q}(t)+g_{u}^{*}(t) \widehat{u}(t)\right) d t \\
=-E \int_{0}^{T}\left\langle m(t) F_{u}^{*}(t)+p(t) G_{u}^{*}(t)+n(t) f_{u}^{*}(t)+g_{u}^{*}(t) \delta(t), \widehat{u}(t)\right\rangle d t .
\end{aligned}
$$

This yields

$$
\begin{aligned}
E\langle n(T), \widehat{y}(T)\rangle-E\langle m(0), \widehat{x}(0)\rangle \\
+E \int_{0}^{T}\left\langle m(t) F_{u}^{*}(t)+p(t) G_{u}^{*}(t)+n(t) f_{u}^{*}(t)+\delta(t) g_{u}^{*}(t), u(t)-u^{*}(t)\right\rangle d t \\
=-E\langle m(T), \widehat{x}(T)\rangle+E\langle n(0), \widehat{y}(0)\rangle \\
=h_{3} E\left\langle\psi_{x}\left(x^{*}(T)\right), \widehat{x}(T)\right\rangle+h_{2} E\left\langle h_{y}\left(y^{*}(0)\right), \widehat{y}(0)\right\rangle \\
\quad+h_{1} E\left\langle\phi_{x}\left(x^{*}(T)\right), \widehat{x}(T)\right\rangle+h_{0} E\left\langle\gamma_{y}\left(y^{*}(0)\right), \widehat{y}(0)\right\rangle \geq 0 .
\end{aligned}
$$


For any $(\xi, \eta, u(\cdot)) \in U$, we get

$$
\begin{aligned}
& E\left\langle n(T), \eta-\eta^{*}\right\rangle-E\left\langle m(0), \xi-\xi^{*}\right\rangle \\
& \quad+E \int_{0}^{T}\left\langle m(t) F_{u}^{*}(t)+p(t) G_{u}^{*}(t)+n(t) f_{u}^{*}(t)+\delta(t) g_{u}^{*}(t), u(t)-u^{*}(t)\right\rangle d t \geq 0 .
\end{aligned}
$$

Thus, it is easy to see that (2.52) holds.

\subsubsection{The General Case}

Define the Hamiltonian

$$
H: \mathbb{R}^{n} \times \mathbb{R}^{n \times l} \times \mathbb{R}^{k} \times \mathbb{R}^{k \times d} \times \mathbb{R}^{n \times d} \times \mathbb{R}^{n} \times \mathbb{R}^{n \times l} \times \mathbb{R}^{k} \times \mathbb{R}^{k \times d} \times[0, T] \longrightarrow \mathbb{R}
$$

by

$$
\begin{aligned}
H(x, z, y, q, u, m, p, n, \delta, t)= & \langle F(t, x, z, y, q, u), m(t)\rangle+\langle G(t, x, z, y, q, u), p(t)\rangle \\
& +\langle f(t, x, z, y, q, u), n(t)\rangle+\langle g(t, x, z, y, q, u), \delta(t)\rangle \\
& +l(x, z, y, q, u, t) .
\end{aligned}
$$

Now we consider the general case where $l(x, z, y, q, u, t) \neq 0, x(x) \neq 0, \lambda(y) \neq 0$.

Since the proof of the maximum principle is essentially similar as in the preceding subsection, we only present the result without proof.

Let $\left(\xi^{*}, \eta^{*}, u^{*}(\cdot)\right)$ be optimal to $(2.17)$ with $\left(x^{*}(\cdot), z^{*}(\cdot), y^{*}(\cdot), q^{*}(\cdot)\right)$ being the corresponding optimal trajectory of (2.13). We define the following adjoint equations:

$$
\begin{gathered}
-d m(t)=-\left(F_{x}^{*}(t) m(t)+G_{x}^{*}(t) p(t)+f_{x}^{*}(t) n(t)+g_{x}^{*}(t) \delta(t)+l_{x}^{*}(t)\right) d t \\
+\left(F_{z}^{*}(t) m(t)+G_{z}^{*}(t) p(t)+f_{z}^{*}(t) n(t)+g_{z}^{*}(t) \delta(t)+l_{z}^{*}(t)\right) d B_{t}-p_{t} d W_{t}, \\
m(T)=-\left(h_{3} \psi_{x}\left(x_{T}^{*}\right)+h_{1} \phi_{x}\left(x_{T}^{*}\right)\right), \\
d n(t)=\left(F_{y}^{*}(t) m(t)+G_{y}^{*}(t) p(t)+f_{y}^{*}(t) n(t)+g_{y}^{*}(t) \delta(t)+l_{y}^{*}(t)\right) d t \\
+\left(F_{q}^{*}(t) m(t)+G_{q}^{*}(t) p(t)+f_{q}^{*}(t) n(t)+g_{q}^{*}(t) \delta(t)+l_{q}^{*}(t)\right) d W_{t}-\delta_{t} d B_{t}, \\
n(0)=h_{2} h_{y}\left(y_{0}^{*}\right)+h_{0} \gamma_{y}\left(y_{0}^{*}\right),
\end{gathered}
$$

where $l_{a}^{*}(t)=l_{a}\left(x^{*}(t), z^{*}(t), y^{*}(t), q^{*}(t), u^{*}(t), t\right), a=x, z, y, q$, respectively. 
Theorem 2.8. We assume (H1) (H3). Let $\left(\xi^{*}, \eta^{*}, u^{*}(\cdot)\right)$ be optimal and let $\left(x^{*}(\cdot), z^{*}(\cdot), y^{*}(\cdot), q^{*}(\cdot)\right)$ be the corresponding optimal trajectory. Then for arbitrary $(\xi, \eta, u(\cdot)) \in U$, one has that the following inequalities hold, for any $t \in[0, T]$ :

$$
\begin{gathered}
\left\langle m(0)-x_{x}\left(\xi^{*}\right), \xi-\xi^{*}\right\rangle \leq 0, \\
\left\langle n(T)+\lambda_{y}\left(\eta^{*}\right), \eta-\eta^{*}\right\rangle \geq 0, \\
\left\langle H_{u}\left(t, x^{*}(t), z^{*}(t), y^{*}(t), q^{*}(t), u^{*}(t), m(t), p(t), n(t), \delta(t)\right), u(t)-u^{*}(t)\right\rangle \geq 0 .
\end{gathered}
$$

Remark 2.9. Let us denote the boundary of $K_{1}$ by $\partial K_{1}$. Set

$$
M \triangleq\left\{w \in \Omega \mid \xi^{*}(\omega) \in \partial K_{1}\right\}
$$

Then

$$
\begin{array}{ll}
m(0)-\chi_{x}\left(\xi^{*}\right) \leq 0 & \text { a.s. on } M, \\
m(0)-\chi_{x}\left(\xi^{*}\right)=0 & \text { a.s. on } M^{c} .
\end{array}
$$

Similar analysis can be used to the boundaries of $K_{2}$ and $K$.

\section{Applications}

In this section, we give three specific cases to illustrate the applications of our obtained results.

\subsection{System Composed of a Forward SDE and a BDSDE}

\section{Classical Formulation}

For given $\xi \in L^{2}\left(\Omega, \mathscr{F}_{0}, P ; \mathbb{R}^{n}\right)$ and $u(\cdot) \in U[0, T]$, we consider the following controlled system composed of a FSDE and a BDSDE:

$$
\begin{gathered}
d y(t)=\bar{b}(t, y(t), u(t)) d t+\sigma(t, y(t), u(t)) d W_{t}, \\
y(0)=b, \\
-d x(t)=\bar{f}(t, x(t), z(t), y(t), u(t)) d t+\bar{g}(t, x(t), z(t), y(t), u(t)) d W_{t}-z(t) d B_{t}, \\
x(0)=\xi,
\end{gathered}
$$

where $b \in R^{k}$ is given, $x(0)=\xi \in K_{1}$, a.s., where $K_{1}$ is a given nonempty convex subset in $R^{n}$. 
Set the mappings

$$
\begin{gathered}
\bar{b}: \Omega \times[0, T] \times \mathbb{R}^{k} \times \mathbb{R}^{n \times d} \longrightarrow \mathbb{R}^{k}, \\
\sigma: \Omega \times[0, T] \times \mathbb{R}^{k} \times \mathbb{R}^{n \times d} \longrightarrow \mathbb{R}^{k \times d}, \\
\bar{f}: \Omega \times[0, T] \times \mathbb{R}^{n} \times \mathbb{R}^{n \times l} \times \mathbb{R}^{k} \times \mathbb{R}^{n \times d} \longrightarrow \mathbb{R}^{n}, \\
\bar{g}: \Omega \times[0, T] \times \mathbb{R}^{n} \times \mathbb{R}^{n \times l} \times \mathbb{R}^{k} \times \mathbb{R}^{n \times d} \longrightarrow \mathbb{R}^{n \times d} .
\end{gathered}
$$

In this case, we regard $u(\cdot)$ and $\xi$ as the control variables. Define the following cost function:

$$
J(\xi, u(\cdot))=E\left[\int_{0}^{T} \bar{l}(t, x(t), z(t), y(t), u(t)) d t+x(\xi)+\lambda(y(T))+\phi(x(T))\right],
$$

where

$$
\begin{aligned}
& \bar{l}: \Omega \times[0, T] \times \mathbb{R}^{n} \times \mathbb{R}^{n \times l} \times \mathbb{R}^{k} \times \mathbb{R}^{n \times d} \longrightarrow \mathbb{R}, \\
& x: \mathbb{R}^{n} \longrightarrow \mathbb{R}, \quad \lambda: \mathbb{R}^{k} \longrightarrow \mathbb{R}, \quad \phi: \mathbb{R}^{n} \longrightarrow \mathbb{R} .
\end{aligned}
$$

We assume

(H1) $\bar{b}, \sigma, \bar{f}, \bar{g}, \bar{l}, x, \lambda$, and $\phi$ are continuous in their arguments and continuously differentiable in $(x, z, y, u)$.

(H2) There exist constants $C>0$ and $0<\alpha<1 / 2$ such that for any $(\omega, t) \in \Omega \times[0, T], u \in$ $\mathbb{R}^{n \times d},\left(x_{1}, z_{1}, y_{1}\right),\left(x_{2}, z_{2}, y_{2}\right) \in \mathbb{R}^{n} \times \mathbb{R}^{n \times l} \times \mathbb{R}^{k}$ the following conditions hold:

$$
\begin{gathered}
\left|\bar{f}\left(t, x_{1}, z_{1}, y_{1}, u\right)-\bar{f}\left(t, x_{2}, z_{2}, y_{2}, u\right)\right|^{2} \leq C\left(\left|x_{1}-x_{2}\right|^{2}+|| z_{1}-z_{2} \|^{2}+\left|y_{1}-y_{2}\right|^{2}\right), \\
\left\|\bar{g}\left(t, x_{1}, z_{1}, y_{1}, u\right)-\bar{g}\left(t, x_{2}, z_{2}, y_{2}, u\right)\right\|^{2} \leq C\left(\left|x_{1}-x_{2}\right|^{2}+\left|y_{1}-y_{2}\right|^{2}\right)+\alpha\left\|z_{1}-z_{2}\right\|^{2} .
\end{gathered}
$$

(H3) The derivatives of $\bar{b}, \sigma, \bar{f}, \bar{g}$ in $(x, y, z, u)$ are bounded; the derivatives of $\bar{l}$ in $(x, y, z, u)$ are bounded by $C(1+|x|+|y|+|z|+\|u\|)$; the derivatives of $x$ and $\phi$ in $x$ are bounded by $C(1+|x|)$; the derivatives of $\lambda$ in $y$ are bounded by $C(1+|y|)$.

Then, for given $\xi \in L^{2}\left(\Omega, \mathcal{F}_{0}, P ; \mathbb{R}^{n}\right)$ and $u(\cdot) \in U[0, T]$, there exists a unique triple

$$
(x(\cdot), y(\cdot), z(\cdot)) \in M^{2}\left(0, T ; \mathbb{R}^{n}\right) \times M^{2}\left(0, T ; \mathbb{R}^{k}\right) \times M^{2}\left(0, T ; \mathbb{R}^{n \times l}\right) .
$$

which solves (3.1).

We assume an additional terminal state constraint $y(T)=\eta \in K_{2}$, a.s., where $K_{2}$ is a given nonempty convex subset in $\mathbb{R}^{k}$. Our stochastic control problem is

$$
\inf J(\xi, u(\cdot))
$$

subject to $u(\cdot) \in U[0, T] ; \quad \xi \in K_{1}$, a.s, $\eta \in K_{2}$, a.s. 


\section{Backward Formulation}

From now on, we give an equivalent backward formulation of the previously mentioned stochastic optimal problem (3.7). To do so we need an additional assumption:

(H4) there exists $\alpha>0$ such that $\left|\sigma\left(y, u_{1}, t\right)-\sigma\left(y, u_{2}, t\right)\right| \geq \alpha\left|u_{1}-u_{2}\right|$ for all $y \in \mathbb{R}^{k}, t \in[0, T]$ and $u_{1}, u_{2} \in \mathbb{R}^{n \times d}$.

Note (H1) and (H4) imply the mapping $u \rightarrow \sigma(y, u, t)$ is a bijection from $\mathbb{R}^{n \times d}$ onto itself for any $(y, t)$.

Let $q \equiv \sigma(y, u, t)$ and denote the inverse function by $u=\tilde{\sigma}(y, q, t)$. Then system (3.1) can be rewritten as

$$
\begin{gathered}
-d y(t)=f(t, y(t), q(t)) d t-q(t) d W_{t}, \\
y(0)=b, \\
-d x(t)=F(t, x(t), z(t), y(t), q(t)) d t+G(t, x(t), z(t), y(t) q(t)) d W_{t}-z(t) d B_{t}, \\
x(0)=\xi,
\end{gathered}
$$

where $f(t, y, q)=-b(t, y, \widetilde{\sigma}(y, q, t))$ and $F(t, x, z, y, q)=\bar{f}(t, x, z, y, \widetilde{\sigma}(y, q, t)), G(t, x, z, y, q)=$ $\bar{g}(t, x, z, y, \widetilde{\sigma}(y, q, t))$.

A key observation that inspires our approach of solving problem (3.7) is that, since $u \rightarrow \sigma(x, u, t)$ is a bijection, $q(\cdot)$ can be regarded as the control; moreover, by the BSDE theory selecting $q(\cdot)$ is equivalent to selecting the terminal value $y(T)$. Hence we introduce the following "controlled" system:

$$
\begin{gathered}
-d y(t)=f(t, y(t), q(t)) d t-q(t) d W_{t}, \\
y(t)=\eta, \\
-d x(t)=F(t, x(t), z(t), y(t), q(t)) d t+G(t, x(t), z(t), y(t), q(t)) d W_{t}-z(t) d B_{t}, \\
x(0)=\xi,
\end{gathered}
$$

where the control variables are the random variables $\xi$ and $\eta$ to be chosen from the following set:

$$
U=\left\{(\xi, \eta) \mid \xi \in K_{1} \text {, a.s. } E|\xi|^{2}<\infty, \eta \in K_{2} \text {, a.s. } E|\eta|^{2}<\infty\right\}
$$

For each $(\xi, \eta) \in U$, consider the following cost:

$$
J(\xi, \eta)=E\left[\int_{0}^{T} l(t, x(t), z(t), y(t), q(t)) d t+x(\xi)+\lambda(\eta)+\phi(x(T))\right]
$$

where $l(t, x, z, y, q)=\bar{l}(t, x, z, y, \widetilde{\sigma}(y, q, t))$. 
This gives rise to the following auxiliary optimization problem:

$$
\begin{gathered}
\inf J(\xi, \eta) \\
\text { subject to }(\xi, \eta) \in U ; y(0)=b,
\end{gathered}
$$

where $y(0)^{(\xi, \eta)}$ is the solution of (3.9) at time 0 under $\xi$ and $\eta$.

It is clear that the original problem (3.7) is equivalent to the auxiliary one (3.12).

Hence, hereafter we focus ourselves on solving (3.12). The advantage of doing this is that, since $\xi$ and $\eta$ now are the control variables, the state constraint in (3.7) becomes a control constraint in (3.12), whereas it is well known in control theory that a control constraint is much easier to deal with than a state constraint. There is, nonetheless, a cost of doing; that is, the original initial condition $y(0)^{(\xi, \eta)}=b$ now becomes a constraint, as shown in (3.12).

From now on, we denote the solution of $(3.9)$ by $\left(x^{(\xi, \eta)}(\cdot), y^{(\xi, \eta)}(\cdot), q^{(\xi, \eta)}(\cdot), z^{(\xi, \eta)}(\cdot)\right)$, whenever necessary, to show the dependence on $(\xi, \eta)$. We also denote $x^{(\xi, \eta)}(0)$ and $y^{(\xi, \eta)}(0)$ by $x(0)^{(\xi, \eta)}$ and $y(0)^{(\xi, \eta)}$, respectively. Finally, it is easy to check that $f, F, G$ and $l$ satisfy similar conditions in Assumptions (H1)-(H3). ing result.

We note that this is a special case of (2.17), so by the same method we have the follow-

\section{Stochastic Maximum Principle}

We define

$$
\begin{gathered}
d n(t)=\left(F_{y}^{*}(t) m(t)+G_{y}^{*}(t) p(t)+f_{y}^{*}(t) n(t)+l_{y}^{*}(t)\right) d t \\
+\left(F_{q}^{*}(t) m(t)+G_{q}^{*}(t) p(t)+f_{q}^{*}(t) n(t)+l_{q}^{*}(t)\right) d W_{t}, \\
n(0)=h_{2}, \\
-d m(t)=-\left(F_{x}^{*}(t) m(t)+G_{x}^{*}(t) p(t)+l_{x}^{*}(t)\right) d t \\
+\left(F_{z}^{*}(t) m(t)+G_{z}^{*}(t) p(t)+l_{z}^{*}(t)\right) d B_{t}-p_{t} d W_{t}, \\
m(T)=-h_{1} \phi_{x}\left(x^{*}(T)\right),
\end{gathered}
$$

where $H_{k}^{*}(t)=H_{k}\left(t, x^{*}(t), z^{*}(t), y^{*}(t), q^{*}(t)\right)(H=F, G, l)$ and $f_{x}^{*}(t)=f_{x}\left(t, x^{*}(t), z^{*}(t)\right), f_{q}^{*}(t)=$ $f_{q}\left(t, x^{*}(t), z^{*}(t)\right), h_{1}$ and $h_{2}$ are defined as in (2.32). It is easy to check that there exist unique processes $n(\cdot)$ and $(m(\cdot), p(\cdot))$ which solve the above equations.

Theorem 3.1. One assumes (H1) (H4). Let $\left(\xi^{*}, \eta^{*}\right)$ be optimal to (3.12), and let $\left(x^{*}(\cdot), y^{*}(\cdot)\right.$, $\left.z^{*}(\cdot), q^{*}(\cdot)\right)$ be the corresponding optimal trajectory. Then for arbitrary $(\xi, \eta) \in U$, one has that the following inequalities hold:

$$
\begin{aligned}
& \left\langle m(0)-\chi_{x}\left(\xi^{*}\right), \xi-\xi^{*}\right\rangle \leq 0, \\
& \left\langle n(T)+\lambda_{y}\left(\eta^{*}\right), \eta-\eta^{*}\right\rangle \geq 0 .
\end{aligned}
$$




\subsection{System Composed of a BDSDE with State Constraints}

Although this case describes a controlled BDSDE system with state constraints, it seems trivial. Thus, we only give a brief illustration. Given $\eta \in L^{2}\left(\Omega, \mathcal{F}_{T}, P ; \mathbb{R}^{k}\right)$ and $u(\cdot) \in U[0, T]$, consider the following BDSDE:

$$
\begin{gathered}
-d y(t)=f(t, y(t), q(t), u(t)) d t+g(t, y(t), q(t), u(t)) d B_{t}-q(t) d W_{t}, \quad 0 \leq t \leq T, \\
y(T)=\eta .
\end{gathered}
$$

For given $u(\cdot) \in U[0, T]$ and $f, g$ satisfying (H2) and (H3), from the Theorem 1.1 in [15], it is easy to check that there exists a unique solution $(y(\cdot), q(\cdot))$ of $(3.15)$.

Note that $y(0)^{(\eta, u(\cdot))}$ is a $\mathcal{F}_{0, T}^{B}$-measurable variable, and

$$
E\left(h\left(y^{(\eta, u(\cdot))}(0)\right)\right)=b
$$

Now we regard $\eta$ and $u(\cdot)$ as the control variables to be chosen from the following set:

$$
U=\left\{(\eta, u(\cdot)) \mid \eta \in K_{1} \text {, a.s., } E\left[|\eta|^{2}\right]<\infty, u(\cdot) \in U[0, T]\right\} .
$$

For each $(\eta, u(\cdot)) \in U$, consider the following cost function:

$$
J(\eta, u(\cdot))=E\left[\int_{0}^{T} l(t, y(t), q(t), u(t)) d t+\lambda(\eta)+\gamma(y(0))\right]
$$

which gives rise to the following optimization problem:

$$
\begin{aligned}
& \inf _{(\eta, u(\cdot)) \in U} J(\eta, u(\cdot)) \\
& \text { subject to } E\left(h\left(y^{(\eta, u(\cdot))}(0)\right)\right)=b .
\end{aligned}
$$

Maximum Principle

Let

$$
H(y, q, u, n, \delta) \triangleq\langle f(t, y, q, u), n(t)\rangle+\langle g(t, y, q, u), \delta(t)\rangle+l(t, y, q, u) .
$$

Then the adjoint equation is

$$
\begin{gathered}
d n(t)=\left(f_{y}^{*}(t) n(t)+g_{y}^{*}(t) \delta(t)+l_{y}^{*}(t)\right) d t-\delta(t) d B_{t}+\left(f_{q}^{*}(t) n(t)+g_{q}^{*}(t) \delta(t)+l_{q}^{*}(t)\right) d W_{t}, \\
n(0)=h_{2} h_{y}\left(y^{*}(0)\right)+h_{0} \gamma_{y}\left(y^{*}(0)\right),
\end{gathered}
$$

where $H_{k}^{*}(t)=H_{k}\left(t, y^{*}(t), q^{*}(t)\right)$ for $H=f, g ; k=y, q$, respectively. 
Theorem 3.2. One assumes (H1) (H3). Let $\eta^{*}$ and $u^{*}(\cdot)$ be the optimal controls and let $\left(y^{*}(\cdot), z^{*}(\cdot)\right)$ be the corresponding optimal trajectory. Then one has

$$
\begin{gathered}
\left\langle n(T)+\lambda_{y}\left(\eta^{*}\right), \eta-\eta^{*}\right\rangle \geq 0, \\
\left\langle H_{u}\left(t, y^{*}, q^{*}, u^{*}, n, \delta\right), u(t)-u^{*}(t)\right\rangle \geq 0 .
\end{gathered}
$$

\subsection{Backward Doubly Stochastic LQ Problem without State Constraints}

Consider the following linear system:

$$
\begin{gathered}
-d x(t)=[A(t) x(t)+B(t) z(t)+C(t) y(t)+D(t) q(t)+E(t) u(t)] d t \\
+\left[A^{\prime}(t) x(t)+B^{\prime}(t) z(t)+C^{\prime}(t) y(t)+D^{\prime}(t) q(t)+E^{\prime}(t) u(t)\right] d W_{t}-z(t) d B_{t}, \\
x(0)=x, \\
-d y(t)=\left[A^{\prime \prime}(t) x(t)+B^{\prime \prime}(t) z(t)+C^{\prime \prime}(t) y(t)+D^{\prime \prime}(t) q(t)+E^{\prime \prime}(t) u(t)\right] d t \\
+\left[A^{\prime \prime \prime}(t) x(t)+B^{\prime \prime \prime}(t) z(t)+C^{\prime \prime \prime}(t) y(t)+D^{\prime \prime \prime}(t) q(t)+E^{\prime \prime \prime}(t) u(t)\right] d B_{t}-q(t) d W_{t}, \\
y(T)=y,
\end{gathered}
$$

where $x$ and $y$ are given constants and $H, H^{\prime}, H^{\prime \prime}, H^{\prime \prime \prime}(H=A, B, C, D$ and $E)$ are corresponding matrices.

The cost function (2.16) becomes

$$
\begin{aligned}
l(t, x(t), z(t), y(t), q(t), u(t))= & \frac{1}{2} F(t) x(t) \cdot x(t)+\frac{1}{2} G(t) z(t) \cdot z(t) \\
& +\frac{1}{2} H(t) y(t) \cdot y(t)+\frac{1}{2} I(t) q(t) \cdot q(t)+\frac{1}{2} J(t) u(t) \cdot u(t), \\
x(x)=0, \quad \lambda(y)= & 0, \quad \phi(x)=\frac{1}{2} U(t) x^{2}, \quad r(y)=\frac{1}{2} Q(t) y^{2},
\end{aligned}
$$

where all functions of $t$ are bounded and $F(t), G(t), H(t), I(t)$ are symmetric non-negative definite, $J(t), U(t), Q(t)$ are symmetric uniformly positive definite.

Then from (2.51), the adjoint equations become

$$
\begin{gathered}
-d m(t)=-\left[A(t) m(t)+A^{\prime}(t) p(t)+A^{\prime \prime}(t) n(t)+A^{\prime \prime \prime}(t) \delta(t)+F(t) x^{*}(t)\right] d t \\
+\left[B(t) m(t)+B^{\prime}(t) p(t)+B^{\prime \prime}(t) n(t)+B^{\prime \prime \prime}(t) \delta(t)+G(t) z^{*}(t)\right] d B_{t}-p(t) d W_{t}, \\
m(T)=-U(T) x^{*}(T), \\
d n(t)=\left[C(t) m(t)+C^{\prime}(t) p(t)+C^{\prime \prime}(t) n(t)+C^{\prime \prime \prime}(t) \delta(t)+H(t) y^{*}(t)\right] d t \\
+\left[D(t) m(t)+D^{\prime}(t) p(t)+D^{\prime \prime}(t) n(t)+D^{\prime \prime \prime}(t) \delta(t)+I(t) q^{*}(t)\right] d W_{t}-\delta(t) d B_{t}, \\
n(0)=Q(0) y^{*}(0) .
\end{gathered}
$$


Define

$$
\begin{aligned}
H(t, x, z, y, q, u, m, p, n, \delta) \triangleq & \langle F(t, x, z, y, q, u), m(t)\rangle+\langle G(t, x, z, y, q, u), p(t)\rangle \\
& +\langle f(t, x, z, y, q, u), n(t)\rangle+\langle g(t, x, z, y, q, u), \delta(t)\rangle \\
& +l(t, x, z, y, q, u) .
\end{aligned}
$$

Suppose that $K$ is an open set. Then we have the following result:

$$
H_{u}\left(t, x^{*}, z^{*}, y^{*}, q^{*}, u^{*}, m, p, n, \delta\right)=E(t) m(t)+E^{\prime}(t) p(t)+E^{\prime \prime}(t) n(t)+E^{\prime \prime \prime}(t) \delta(t)+J(t) u(t)=0 .
$$

Thus,

$$
u^{*}(t)=J^{-1}(t)\left[E(t) m(t)+E^{\prime}(t) p(t)+E^{\prime \prime}(t) n(t)+E^{\prime \prime \prime}(t) \delta(t)\right]
$$

However, the maximum principle gives only the necessary condition for optimal control. We also have the following theorem.

Theorem 3.3. The function $u^{*}(t)=J^{-1}(t)\left[E(t) m(t)+E^{\prime}(t) p(t)+E^{\prime \prime}(t) n(t)+E^{\prime \prime \prime}(t) \delta(t)\right]$ is the unique optimal control for backward doubly stochastic LQ problems, where $\left(x^{*}(\cdot), z^{*}(\cdot), y^{*}(\cdot), q^{*}(\cdot)\right)$ and $(m(\cdot), p(\cdot), n(\cdot), \delta(\cdot))$ are solutions of above equations.

Proof. First let us prove that the $u^{*}(\cdot)$ is the optimal control. For all $v(\cdot) \in K$, let $\left(x^{v}(\cdot), z^{v}(\cdot), y^{v}(\cdot), q^{v}(\cdot)\right)$ be the corresponding trajectory of (3.23), we get

$$
\begin{aligned}
& J(v(\cdot))-J\left(u^{*}(\cdot)\right) \\
& =\frac{1}{2}\left\{\int _ { 0 } ^ { T } \left[\left\langle F(t) x^{v}(t), x^{v}(t)\right\rangle-\left\langle F(t) x^{*}(t), x^{*}(t)\right\rangle+\left\langle G(t) z^{v}(t), z^{v}(t)\right\rangle\right.\right. \\
& -\left\langle G(t) z^{*}(t), z^{*}(t)\right\rangle+\left\langle H(t) y^{v}(t), y^{v}(t)\right\rangle-\left\langle H(t) y^{*}(t), y^{*}(t)\right\rangle+\left\langle I(t) q^{v}(t), q^{v}(t)\right\rangle \\
& \left.-\left\langle I(t) q^{*}(t), q^{*}(t)\right\rangle+\langle J(t) v(t), v(t)\rangle-\left\langle J(t) u^{*}(t), u^{*}(t)\right\rangle\right] d t \\
& +\left\langle U(T) x^{v}(T), x^{v}(T)\right\rangle \\
& \left.-\left\langle U(T) x^{*}(T), x^{*}(T)\right\rangle+\left\langle Q(0) y^{v}(0), y^{v}(0)\right\rangle-\left\langle Q(0) y^{*}(0), y^{*}(0)\right\rangle\right\} \\
& \geq E\left\{\int _ { 0 } ^ { T } \left[\left\langle F(t) x^{*}(t), x^{v}(t)-x^{*}(t)\right\rangle+\left\langle G(t) z^{*}(t), z^{v}(t)-z^{*}(t)\right\rangle\right.\right. \\
& \left.+\left\langle H(t) y^{*}(t), y^{v}(t)-y^{*}(t)\right\rangle+\left\langle I(t) q^{*}(t), q^{v}(t)-q^{*}(t)\right\rangle+\left\langle J(t) u^{*}(t), v(t)-u^{*}(t)\right\rangle\right] d t \\
& \left.+\left\langle U(T) x^{*}(T), x^{v}(T)-x^{*}(T)\right\rangle+\left\langle Q(0) y^{*}(0), y^{v}(0)-y^{*}(0)\right\rangle\right\} .
\end{aligned}
$$


Using Lemma 2.1 to $\left\langle x^{v}(t)-x^{*}(t), m(t)\right\rangle+\left\langle y^{v}(t)-y^{*}(t), n(t)\right\rangle$, we obtain

$$
\begin{aligned}
\left\langle U(T) x^{*}(T), x^{v}(T)-x^{*}(T)\right\rangle+\left\langle Q(0) y^{*}(0), y^{v}(0)-y^{*}(0)\right\rangle \\
=-E \int_{0}^{T}\left[\left\langle F(t) x^{*}(t), x^{v}(t)-x^{*}(t)\right\rangle+\left\langle G(t) z^{*}(t), z^{v}(t)-z^{*}(t)\right\rangle\right. \\
\quad+\left\langle H(t) y^{*}(t), y^{v}(t)-y^{*}(t)\right\rangle+\left\langle I(t) q^{*}(t), q^{v}(t)-q^{*}(t)\right\rangle\left\langle J(t) u^{*}(t), v(t)-u^{*}(t)\right\rangle \\
\quad+\left\langle E(t) m(t), v(t)-u^{*}(t)\right\rangle+\left\langle E^{\prime}(t) p(t), v(t)-u^{*}(t)\right\rangle+\left\langle E^{\prime \prime}(t) n(t), v(t)-u^{*}(t)\right\rangle \\
\left.+\left\langle E^{\prime \prime \prime}(t) \delta(t), v(t)-u^{*}(t)\right\rangle\right] d t .
\end{aligned}
$$

So by the definition of $u^{*}(t)$,

$$
\begin{aligned}
& J(v(\cdot))-J\left(u^{*}(\cdot)\right) \\
& \qquad E \int_{0}^{T}\left[\left\langle E(t) m(t), v(t)-u^{*}(t)\right\rangle+\left\langle E^{\prime}(t) p(t), v(t)-u^{*}(t)\right\rangle+\left\langle E^{\prime \prime}(t) n(t), v(t)-u^{*}(t)\right\rangle\right. \\
& \left.\quad+\left\langle E^{\prime \prime \prime}(t) \delta(t), v(t)-u^{*}(t)\right\rangle+\left\langle J(t) u^{*}(t), v(t)-u^{*}(t)\right\rangle\right] d t=0 .
\end{aligned}
$$

From the arbitrariness of $v(\cdot) \in K$, we deduce $u^{*}(t)$ is the optimal control.

The proof of the uniqueness of the optimal control is classical. Assume that $u^{1}(\cdot)$ and $u^{2}(\cdot)$ are both optimal controls, and the corresponding trajectories are $\left(x^{1}(\cdot), z^{1}(\cdot), y^{1}(\cdot), q^{1}(\cdot)\right)$ and $\left(x^{2}(\cdot), z^{2}(\cdot), y^{2}(\cdot), q^{2}(\cdot)\right)$. By the uniqueness of solutions of (3.23), we know that the trajectory corresponding to $\left(u^{1}(\cdot)+u^{2}(\cdot)\right) / 2$ is $\left(\left(x^{1}(\cdot)+x^{2}(\cdot)\right) / 2,\left(z^{1}(\cdot)+z^{2}(\cdot)\right) / 2,\left(y^{1}(\cdot)+\right.\right.$ $\left.\left.y^{2}(\cdot)\right) / 2,\left(q^{1}(\cdot)+q^{2}(\cdot)\right) / 2\right)$, and notice that $J(t), U(t), Q(t)$ are positive, $F(t), G(t), H(t), I(t)$ are nonnegative, we have

$$
\begin{gathered}
J\left(u^{1}(\cdot)\right)=J\left(u^{2}(\cdot)\right)=\alpha \geq 0, \\
2 \alpha=J\left(u^{1}(\cdot)\right)+J\left(u^{2}(\cdot)\right) \\
\geq 2 J\left(\frac{u^{1}(\cdot)+u^{2}(\cdot)}{2}\right)+2 E \int_{0}^{T}\left\langle J(t) \frac{u^{1}(\cdot)-u^{2}(\cdot)}{2}, \frac{u^{1}(\cdot)-u^{2}(\cdot)}{2}\right\rangle d t \\
\geq 2 \alpha+\frac{\beta}{2} E \int_{0}^{T}\left|u^{1}(\cdot)-u^{2}(\cdot)\right|^{2} d t,
\end{gathered}
$$

here $\beta>0$. So

$$
E \int_{0}^{T}\left|u^{1}(\cdot)-u^{2}(\cdot)\right|^{2} d t \leq 0
$$

which shows that $u^{1}(\cdot)=u^{2}(\cdot)$. 
Example 3.4. Consider the following backward doubly stochastic LQ problem, where $t \in$ $[0,1], u(\cdot) \in U[0,1]=[-1,1]$ and $n=k=d=l=1$ :

$$
\begin{gathered}
-d x(t)=\frac{1}{2}(y(t)+2 u(t)) d W_{t}-z(t) d B_{t}, \\
x(0)=0 \\
-d y(t)=\frac{1}{2}(x(t)+u(t)) d B_{t}-q(t) d W_{t} \\
y(1)=0 .
\end{gathered}
$$

We want to minimize the following cost function:

$$
J(u(\cdot))=E \int_{0}^{1}\left(x(s)^{2}-y(s)^{2}+z(s)^{2}-q(s)^{2}+2 x(s) u(s)-4 y(s) u(s)\right) d s+E x(1)^{2}+E y(0)^{2}
$$

From (3.34), we get, for $t \in[0,1]$,

$$
\begin{gathered}
x(t)=-\int_{0}^{t}(y(s)+2 u(s)) d W_{s}+\int_{0}^{t} z(s) d B_{s}, \\
y(t)=\int_{t}^{1}(x(s)+u(s)) d B_{s}-\int_{t}^{1} q(s) d W_{s} .
\end{gathered}
$$

By substituting $x(\cdot)$ and $y(\cdot)$ into the cost function, we obtain

$$
J(u(\cdot))=E \int_{0}^{1} 3 u(s)^{2} d s
$$

Thus, the optimal control is $u^{*}(t) \equiv 0, t \in[0,1]$ with the optimal state trajectory

$$
\left(x^{*}(t), z^{*}(t), y^{*}(t), q^{*}(t)\right) \equiv 0, \quad t \in[0,1]
$$

The adjoint equations are

$$
\begin{aligned}
d m(t)= & n(t) d t+p(t) d W_{t}, \\
& m(1)=0, \\
d n(t)= & p(t) d t-\delta(t) d B_{t}, \\
& n(0)=0 .
\end{aligned}
$$

It is obvious that $(m(\cdot), p(\cdot), n(\cdot), \delta(\cdot))=(0,0,0,0)$ is the unique solution of the above equation. 


\section{Acknowledgments}

The authors would like to thank Professor Shige Peng for some useful conversations. This work was supported by National Natural Science Foundation of China (no. 11171187, no. 10871118, and no. 10921101); supported by the Programme of Introducing Talents of Discipline to Universities of China (no. B12023); supported by Program for New Century Excellent Talents in University of China.

\section{References}

[1] N. El Karoui, S. Peng, and M. C. Quenez, "Backward stochastic differential equations in finance," Mathematical Finance, vol. 7, no. 1, pp. 1-71, 1997.

[2] É. Pardoux and S. G. Peng, "Adapted solution of a backward stochastic differential equation," Systems E Control Letters, vol. 14, no. 1, pp. 55-61, 1990.

[3] F. Antonelli, "Backward-forward stochastic differential equations," The Annals of Applied Probability, vol. 3, no. 3, pp. 777-793, 1993.

[4] Y. Hu and S. Peng, "Solution of forward-backward stochastic differential equations," Probability Theory and Related Fields, vol. 103, no. 2, pp. 273-283, 1995.

[5] J. Ma, P. Protter, and J. M. Yong, "Solving forward-backward stochastic differential equations explicitly-a four step scheme," Probability Theory and Related Fields, vol. 98, no. 3, pp. 339-359, 1994.

[6] J. Ma and J. Yong, Forward-Backward Stochastic Differential Equations and Their Applications, vol. 1702 of Lecture Notes in Mathematics, Springer, Berlin, Germany, 1999.

[7] J. Yong, "Finding adapted solutions of forward-backward stochastic differential equations: method of continuation," Probability Theory and Related Fields, vol. 107, no. 4, pp. 537-572, 1997.

[8] Y. Hu, "N-person differential games governed by semilinear stochastic evolution systems," Applied Mathematics and Optimization, vol. 24, no. 3, pp. 257-271, 1991.

[9] J. Yong and X. Y. Zhou, Stochastic Controls Hamiltonian Systems and HJB Equations, vol. 43, Springer, New York, NY, USA, 1999.

[10] R. Buckdahn and Y. Hu, "Hedging contingent claims for a large investor in an incomplete market," Advances in Applied Probability, vol. 30, no. 1, pp. 239-255, 1998.

[11] J. Cvitanić and J. Ma, "Hedging options for a large investor and forward-backward SDE's," The Annals of Applied Probability, vol. 6, no. 2, pp. 370-398, 1996.

[12] Y. Hu, "Potential kernels associated with a filtration and forward-backward SDEs," Potential Analysis, vol. 10, no. 2, pp. 103-118, 1999.

[13] P. Briand and Y. Hu, "Probabilistic approach to singular perturbations of semilinear and quasilinear parabolic PDEs," Nonlinear Analysis. Theory, Methods \& Applications A, vol. 35, no. 7, pp. 815-831, 1999.

[14] R. Buckdahn and Y. Hu, "Probabilistic approach to homogenizations of systems of quasilinear parabolic PDEs with periodic structures," Nonlinear Analysis. Theory, Methods \& Applications A, vol. 32, no. 5, pp. 609-619, 1998.

[15] E. Pardoux and S. Peng, "Backward doubly stochastic differential equations and systems of quasilinear SPDEs," Probability Theory and Related Fields, vol. 98, no. 2, pp. 209-227, 1994.

[16] S. Peng and Y. Shi, "A type of time-symmetric forward-backward stochastic differential equations," Comptes Rendus Mathématique, vol. 336, no. 9, pp. 773-778, 2003.

[17] Y. Han, S. Peng, and Z. Wu, "Maximum principle for backward doubly stochastic control systems with applications," SIAM Journal on Control and Optimization, vol. 48, no. 7, pp. 4224-4241, 2010.

[18] A. Bensoussan, Stochastic Control by Functional Analysis Methods, vol. 11, North-Holland, Amsterdam, The Netherlands, 1982.

[19] S. Ji, "Dual method for continuous-time Markowitz's problems with nonlinear wealth equations," Journal of Mathematical Analysis and Applications, vol. 366, no. 1, pp. 90-100, 2010.

[20] S. Ji and S. Peng, "Terminal perturbation method for the backward approach to continuous time mean-variance portfolio selection," Stochastic Processes and their Applications, vol. 118, no. 6, pp. 952 $967,2008$.

[21] S. Ji and X. Y. Zhou, "A maximum principle for stochastic optimal control with terminal state constraints, and its applications," Communications in Information and Systems, vol. 6, no. 4, pp. 321-337, 2006. 
[22] S. Ji and X. Y. Zhou, "A generalized Neyman-Pearson lemma for g-probabilities," Probability Theory and Related Fields, vol. 148, no. 3-4, pp. 645-669, 2010.

[23] T. R. Bielecki, H. Jin, S. R. Pliska, and X. Y. Zhou, "Continuous-time mean-variance portfolio selection with bankruptcy prohibition," Mathematical Finance, vol. 15, no. 2, pp. 213-244, 2005.

[24] N. El Karoui, S. Peng, and M. C. Quenez, "A dynamic maximum principle for the optimization of recursive utilities under constraints," The Annals of Applied Probability, vol. 11, no. 3, pp. 664-693, 2001.

[25] J. Yong, "Forward-backward stochastic differential equations with mixed initial-terminal conditions," Transactions of the American Mathematical Society, vol. 362, no. 2, pp. 1047-1096, 2010.

[26] J. Yong, “Optimality variational principle for controlled forward-backward stochastic differential equations with mixed initial-terminal conditions," SIAM Journal on Control and Optimization, vol. 48, no. 6, pp. 4119-4156, 2010.

[27] D. Nualart and É. Pardoux, "Stochastic calculus with anticipating integrands," Probability Theory and Related Fields, vol. 78, no. 4, pp. 535-581, 1988.

[28] I. Ekeland, "On the variational principle," Journal of Mathematical Analysis and Applications, vol. 47, pp. 324-353, 1974. 


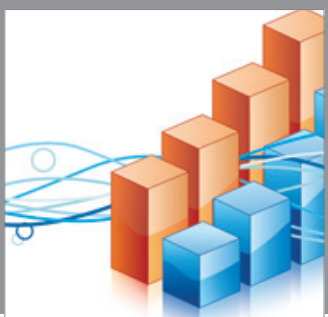

Advances in

Operations Research

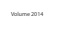

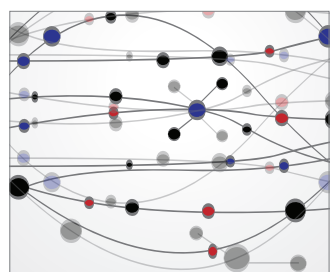

\section{The Scientific} World Journal
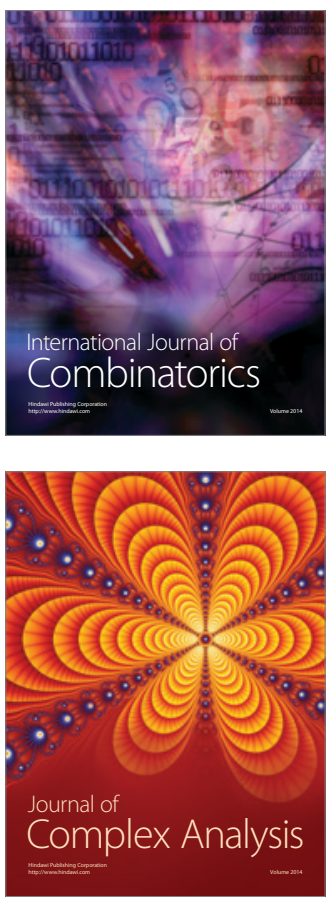

International Journal of

Mathematics and

Mathematical

Sciences
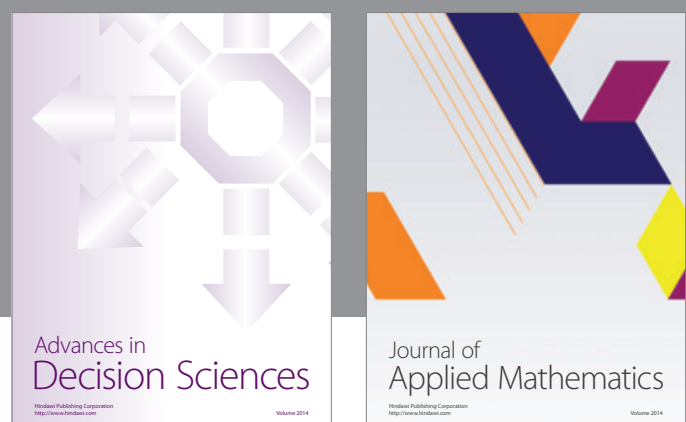

Journal of

Applied Mathematics
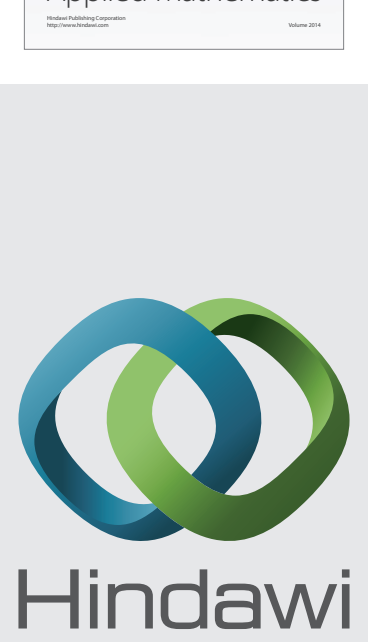

Submit your manuscripts at http://www.hindawi.com
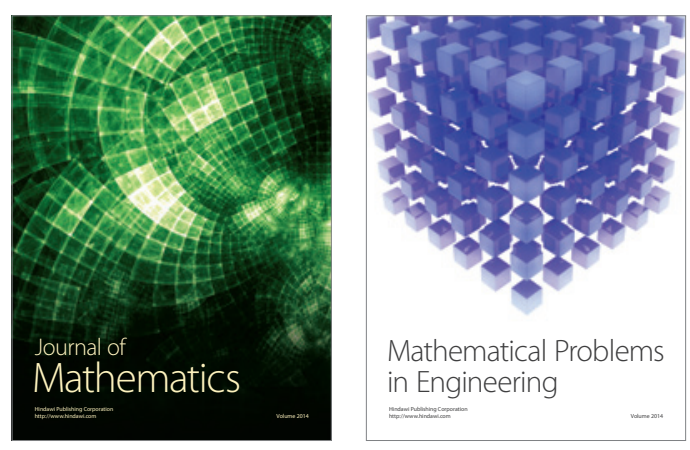

Mathematical Problems in Engineering
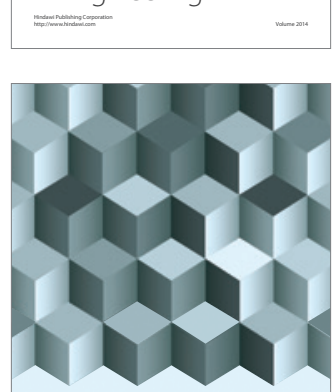

Journal of

Function Spaces
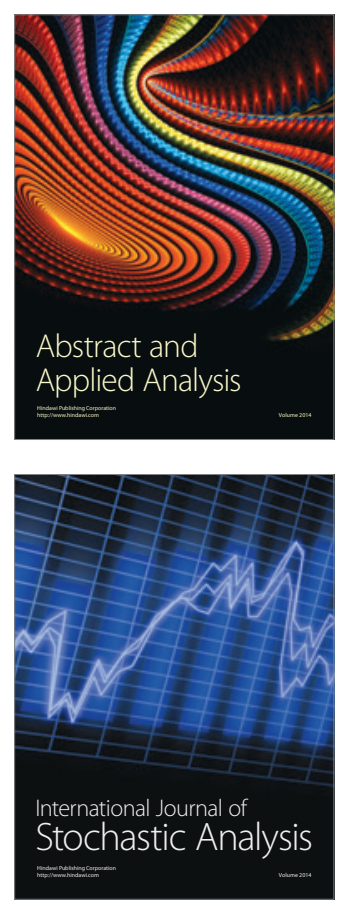

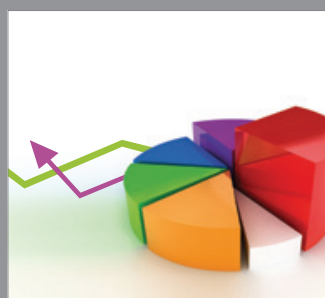

ournal of

Probability and Statistics

Promensencen
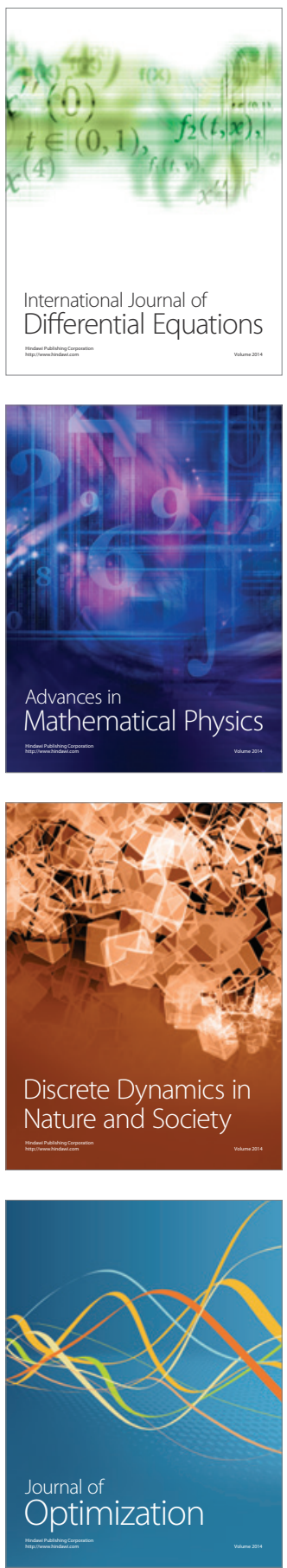